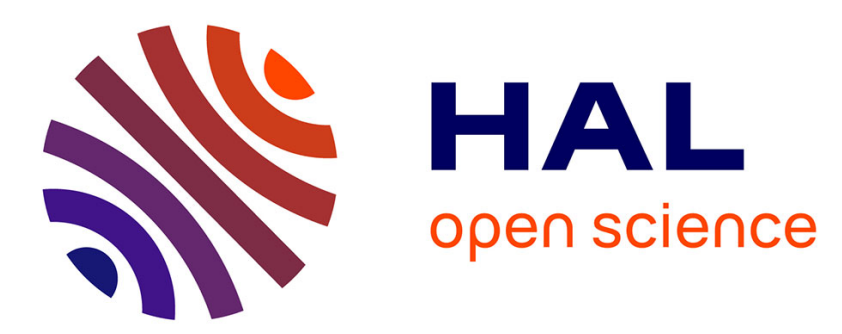

\title{
Radiative heat transfer and radiative thermal energy for two-dimensional nanoparticle ensembles
}

\author{
Minggang Luo, Junming Zhao, Linhua Liu, Mauro Antezza
}

\section{To cite this version:}

Minggang Luo, Junming Zhao, Linhua Liu, Mauro Antezza. Radiative heat transfer and radiative thermal energy for two-dimensional nanoparticle ensembles. Physical Review B, 2020, 102, pp.024203. 10.1103/PhysRevB.102.024203 . hal-02908106

\section{HAL Id: hal-02908106 https://hal.science/hal-02908106}

Submitted on 14 Oct 2020

HAL is a multi-disciplinary open access archive for the deposit and dissemination of scientific research documents, whether they are published or not. The documents may come from teaching and research institutions in France or abroad, or from public or private research centers.
L'archive ouverte pluridisciplinaire HAL, est destinée au dépôt et à la diffusion de documents scientifiques de niveau recherche, publiés ou non, émanant des établissements d'enseignement et de recherche français ou étrangers, des laboratoires publics ou privés. 


\title{
Radiative heat transfer and radiative thermal energy for two-dimensional nanoparticle ensembles
}

\author{
Minggang Luo $\odot,{ }^{1,2}$ Junming Zhao, ${ }^{1,3, *}$ Linhua Liu $\odot,{ }^{4}$ and Mauro Antezza $\odot^{2,5, \dagger}$ \\ ${ }^{1}$ School of Energy Science and Engineering, Harbin Institute of Technology, 92 West Street, Harbin 150001, China \\ ${ }^{2}$ Laboratoire Charles Coulomb (L2C) UMR 5221 CNRS-Université de Montpellier, F- 34095 Montpellier, France \\ ${ }^{3}$ Key Laboratory of Aerospace Thermophysics, Ministry of Industry and Information Technology, Harbin 150001, China \\ ${ }^{4}$ School of Energy and Power Engineering, Shandong University, Qingdao 266237, China \\ ${ }^{5}$ Institut Universitaire de France, 1 rue Descartes, F-75231 Paris Cedex 05, France
}

(Received 28 April 2020; revised 19 June 2020; accepted 6 July 2020; published 21 July 2020)

\begin{abstract}
Radiative heat transfer (RHT) and radiative thermal energy (RTE) for two-dimensional (2D) nanoparticle ensembles are investigated in the framework of many-body radiative heat transfer theory. We consider nanoparticles made of different materials: metals $(\mathrm{Ag})$, polar dielectrics $(\mathrm{SiC})$, or insulator-metallic phase-change materials $\left(\mathrm{VO}_{2}\right)$. We start by investigating the RHT between two parallel 2D finite-size square-lattice nanoparticle ensembles, with particular attention to many-body interactions (MBI) effects. We fix the particle radius $(a)$ as the smallest length scale, and we describe the electromagnetic scattering from particles within the dipole approximation. Depending on the minimal distance between the in-plane particles (the lattice spacing $p$ for periodic systems), on the separation $d$ between the two lattice and on the thermal wavelength $\lambda_{T}=\hbar c / k_{B} T$, we systematically analyze the different physical regimes characterizing the RHT. Four regimes are identified, rarefied regime, dense regime, non-MBI regime, and $\mathrm{MBI}$ regime, respectively. When $p \ll \lambda_{T}$, a multiple scattering of the electromagnetic field inside the systems gives rise to a MBI regime. MBI effects manifest themselves in different ways, depending on the separation $d$ : (a) If $d>\lambda_{T}$, due to the pure intra-ensemble MBI inside each 2D ensemble, the total heat conductance is less affected, and the thermal conductance spectrum manifests a single peak which is nonetheless shifted with respect to the one typical of two isolated nanoparticles. (b) If $d<\lambda_{T}$, there is a strong simultaneous intra-ensemble and inter-ensemble MBI. In this regime there is a direct quantitative effect on the heat conductance, in addition to a qualitative effect on the thermal conductance spectrum, which now manifests a new second peak. As for the RTE, to correctly describe the radiation emitted by metallic nanoparticles, we derive an expression of the Poynting vector including also magnetic contribution, in addition to the electric one. By analyzing both periodic and nonperiodic ensembles, we show that the RTE emitted by a single 2D nanoparticle ensemble is sensitive to the particle distribution. As instance, we see that the RTE emitted by 2D concentric-ring-configuration ensemble has an inhibition feature near the center of the ensemble.
\end{abstract}

DOI: 10.1103/PhysRevB.102.024203

\section{INTRODUCTION}

Near-field radiative heat transfer (NFRHT) has recently attracted much attention for both fundamental and applicative reasons. When the separation distance between two objects is comparable to or less than the thermal wavelength $\lambda_{T}=$ $\hbar c / k_{B} T$, near-field (evanescent waves) effect plays a dominant role in determining the net radiative heat exchange. The fluctuational electrodynamics theory developed by Rytov et al. [1] is the basic theoretical framework to analyze NFRHT. NFRHT between two planar surfaces [2-8], two isolated nanoparticles [9-11], two spheres [12], one dipole and surface [13], two nanoparticles above a substrate [14-17], and between two nanoparticles separated by a multilayer plate [18] were investigated theoretically recently in such framework. The theory has been set in a general framework, and it is now possible to calculate the NFRHT between two or many

\footnotetext{
*jmzhao@hit.edu.cn

†mauro.antezza@umontpellier.fr
}

bodies of arbitrary shape and dielectric permittivity using a general scattering matrix method $[19,20]$, which was then applied to the many-body system with planar geometry [21]. On the experimental side, the radiative heat flux between two objects (e.g., two plates [22-26], one plate, and one sphere or tip [27-29]) has been experimentally proven to be several orders of magnitude larger than the Planck's black-body limit due to evanescent wave tunneling.

In this paper we consider the radiative heat transfer (RHT) between ensembles of nanoparticles. NFRHT in dense nanoparticle systems is difficult to determine due to the complex many-body interaction [30,31]. In a dense nanoparticle system, nanoparticles lie in the near field of each other, which leads to the significant multiple scattering of the thermally excited evanescent wave, hence, the many-body interaction (MBI) will play a key role, and the two-body framework cannot be directly applied. To analyze NFRHT in systems composed of many nanoparticles, many theoretical frameworks have been developed, e.g., the many-body radiative heat transfer theory [32,33], scattering matrix method [19,20], trace formulas method [34,35], and the quasianalytic solution 
[36] based on the framework proposed to investigate NFRHT between two spheres [12].

For NFRHT in the three-dimensional (3D) nanoparticle system, some important progress has been reported recently. The MBI can not only enhance, but also inhibit, and even have nearly no effect on the radiative heat flux between dielectric many-particle systems [32,37], metallic many-nanoparticle clusters [38] and core-shell nanoparticle assemblies [39], as well as the one-dimensional (1D) nanoparticle chains [40]. By investigating NFRHT in the dense particle system from the point of view of continuum medium, the heat superdiffusion was found in the plasmonic nanostructure networks due to NFRHT based on the fractional diffusion theory [30]. Then, a similar heat superdiffusion was found in the periodically arranged planar SiC plates [41]. Recently, a new method was developed to calculate the diffusive radiative thermal conductivity of arbitrary collections of nanoparticles [42], which is an important progress relative to the kinetic method used to calculate the effective radiative thermal conductivity of 1D nanoparticle chain [43-45]. Also, the radiative thermal energy (RTE) emitted in the near field by a set of interacting nanoparticles has been the object of investigations, and has been recently predicted to focus the field in spots that are much smaller than those obtained with a single thermal source [46].

For NFRHT in the two-dimensional (2D) nanoparticle ensembles, some interesting phenomena have been reported. On the one hand, the inter-ensemble NFRHT between the 2D nanoparticle ensembles has been investigated in the extreme near field. The NFRHT between two gold nanoparticle array layers with 1-nm separation layer edge to layer edge was reported [47], where the multipole contribution to NFRHT should be considered. An interesting oscillatorylike feature of the NFRHT with translation of one array along its extending direction was observed. On the other hand, the investigation on intra-ensemble NFRHT in the 2D fractal nanoparticle ensembles also have been reported and the spatial distribution of nanoparticles in a 2D nanoparticle ensemble was demonstrated to play a key role in determining the radiative heat flux [48].

As for the RHT and radiative thermal energy for the 2D nanoparticle ensembles, there are several important aspects still needing investigation. Indeed, the way in which the MBI manifests itself in the total thermal conductance and in the thermal conductance spectrum has not been investigated. A study of the combined effects coming from different 2D geometrical arrangements (periodic, nonperiodic, concentric rings) and dielectric properties (metals, polar dielectrics, metal-insulator phase-change materials) of the nanoparticle ensembles is missing. We address these points in this paper, where the RHT between 2D nanoparticle ensembles is investigated by means of many-body radiative heat transfer theory [32], further extended to metallic system in the coupled electric and magnetic dipole (CEMD) approach [33]. We also study, for these systems, the RTE.

This work is organized as follows. In Sec. II, the CEMD approach is presented in brief, together with the expression for the RHT and thermal conductance. Concerning the RTE, the expression of the Poynting vector is derived for the general case where also magnetic dipoles are present, in addition to

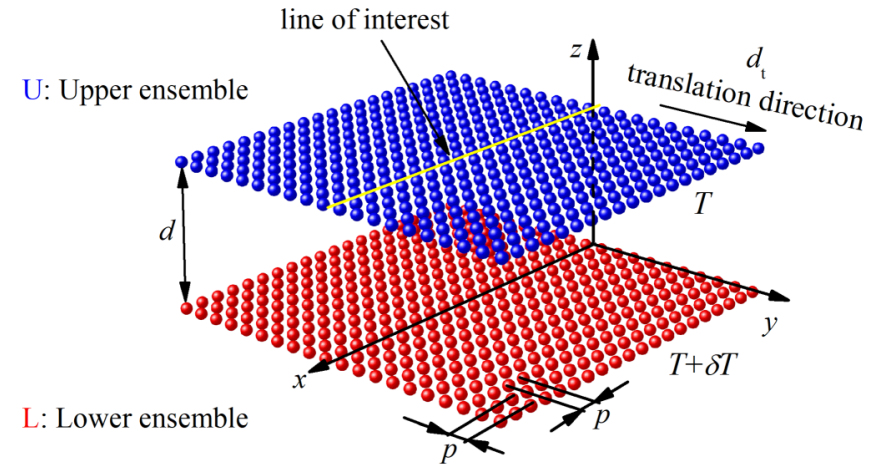

FIG. 1. Schematic of the 2D finite-size square-lattice nanoparticle ensembles. RHT between the upper nanoparticle ensemble (U, at temperature $T$ ) and the lower nanoparticle ensemble $(\mathrm{L}$, at temperature $T+\delta T$ ) is investigated. Nanoparticle radius is $a$. The separation distance between the ensemble $\mathrm{L}$ and $\mathrm{U}$ center to center is $d . d_{\mathrm{t}}$ is the translation distance of the ensemble $\mathrm{U}$ relative to the ensemble L. Periodicity of the periodically distributed nanoparticle ensemble is $p$. $N$ is the number of nanoparticles in each ensemble.

electric ones. This extension of the existing Poynting vector expression allows to describe the thermal emission from both dielectric and metallic nanoparticles. In addition, the physical model of the 2D nanoparticle ensemble and optical properties of the materials used in this work are also given. In Sec. III, asymptotic regimes of RHT between 2D finite-size squarelattice nanoparticle ensembles are summarized, as well as the simplified formulas for the thermal conductance in different regimes. In Sec. IV, RHT between 2D periodic nanoparticle ensembles are analyzed, with particular attention devoted to MBI effects. The effects of metal-insulator phase change of the nanoparticles and of the lateral translation of the two parallel 2D ensembles are also analyzed. In Sec. V, RTE emitted by the single 2D nanoparticle ensemble is analyzed, as a function of the particle distribution (e.g., periodic, random, and concentric-ring configurations) and dielectric properties. A study of the relative weight of the magnetic dipole and electric dipole contributions to RTE is also conducted.

\section{THEORETICAL MODELS}

In this section, we describe the physical systems, the theoretical models for the RHT and RTE, and finally the optical properties of the materials we use.

\section{A. Physical systems: 2D nanoparticle ensembles}

In this paper we will investigate RHT between 2D nanoparticle ensembles by studying radiative thermal conductance between two parallel 2D finite-size square-lattice nanoparticle ensembles (Sec. IV) and RTE emitted by a single 2D periodic and nonperiodic nanoparticle ensemble (Sec. V). We will hence introduce two physical systems, respectively.

Concerning the RHT, we will focus on parallel 2D finitesize square-lattice nanoparticle ensembles, as shown in Fig. 1.

The radiative thermal conductance between the lower ensemble (L, at temperature $T+\delta T$ ) and upper ensemble (U, at temperature $T$ ) is calculated with various separation distance $d$ center to center. In general, each ensemble is composed of 


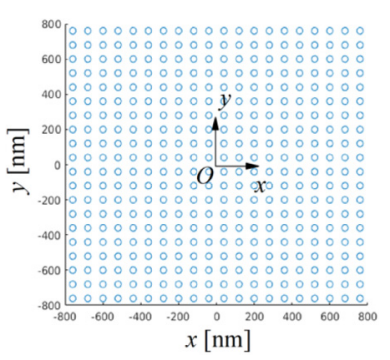

(a) Periodically distributed particle ensemble

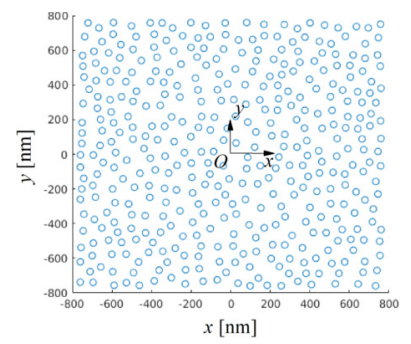

(b) Randomly distributed particle ensemble

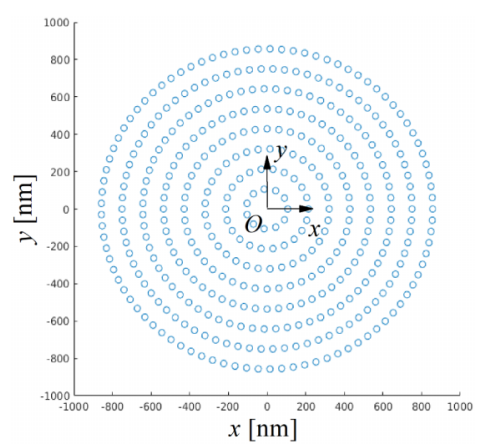

(c) Concentric ring configuration particle ensemble

FIG. 2. Three kinds of nanoparticle distribution: (a) periodically distributed particle ensemble; (b) randomly distributed particle ensemble; and (c) concentric-ring-configuration particle ensemble. $N=400, a=20 \mathrm{~nm}$.

$N=400$ nanoparticles. The nanoparticle radius is $a$. When needed, we will evaluate the energy density and Poynting vector along the yellow line shown in the Fig. 1. When investigating the effect of translation of particle ensemble on RHT, the lower ensemble is fixed and the upper ensemble is translated along the translation direction. The origin of the Cartesian coordinate system is fixed at the center of an edge of the lower ensemble and the particle ensemble is periodically distributed $20 \times 20$ nanoparticles ensemble. The periodicity $p$ is the separation distance between two neighboring particles center to center in the line parallel to the edge of the ensemble.

When we discuss the RTE emitted by the single nanoparticle ensemble (Sec. V), we will refer to the physical systems of Fig. 2, where three different kinds of 2D nanoparticle distributions are considered: (a) periodic ensemble, (b) random ensemble, and (c) concentric-ring configuration. The origin of the Cartesian coordinate system is set at the center of the ensemble. We will consider $N=400$ nanoparticles of radius $a=20 \mathrm{~nm}$.

\section{B. Radiative heat transfer and thermal conductance between nanoparticle ensembles}

Let us start considering two particles. In the framework of the CEMD approach, the power absorbed by the $i$ th particle and radiated by $j$ th particle is written in the Landauer formalism $[32,33,38]$

$$
\varphi_{j \rightarrow i}=3 \int_{0}^{+\infty} \frac{d \omega}{2 \pi} \Theta\left(\omega, T_{j}\right) \mathcal{T}_{i, j}(\omega),
$$

where $\omega$ is electromagnetic field angular frequency, $\Theta\left(\omega, T_{j}\right)$ is the mean energy of the harmonic Planck's oscillator, and $\mathcal{T}_{i, j}(\omega)$ is the transmission coefficient between the $j$ th and $i$ th particles, given by $[33,38]$

$$
\begin{aligned}
\mathcal{T}_{i, j}(\omega)= & \frac{4}{3} k^{4}\left[\operatorname{Im}\left(\chi_{E}^{i}\right) \operatorname{Im}\left(\chi_{E}^{j}\right) \operatorname{Tr}\left(G_{i j}^{E E} G_{i j}^{E E \dagger}\right)\right. \\
& +\operatorname{Im}\left(\chi_{E}^{i}\right) \operatorname{Im}\left(\chi_{H}^{j}\right) \operatorname{Tr}\left(G_{i j}^{E M} G_{i j}^{E M \dagger}\right) \\
& +\operatorname{Im}\left(\chi_{H}^{i}\right) \operatorname{Im}\left(\chi_{E}^{j}\right) \operatorname{Tr}\left(G_{i j}^{M E} G_{i j}^{M E \dagger}\right) \\
& \left.+\operatorname{Im}\left(\chi_{H}^{i}\right) \operatorname{Im}\left(\chi_{H}^{j}\right) \operatorname{Tr}\left(G_{i j}^{M M} G_{i j}^{M M \dagger}\right)\right] .
\end{aligned}
$$

Here, parameters $\chi_{E}=\alpha_{E}-\frac{i k^{3}}{6 \pi}\left|\alpha_{E}\right|^{2}$ and $\chi_{H}=\alpha_{H}-$ $\frac{i k^{3}}{6 \pi}\left|\alpha_{H}\right|^{2}$ are introduced [10], $\alpha_{E}$ and $\alpha_{H}$ are electric and magnetic dipole polarizabilities, $k$ is the wave vector in vacuum, $G_{i j}^{\nu \tau}(\nu, \tau=E$ or $M)$ is the Green's function for the many-particle system considering the many-body interaction, which is the solution of Eq. (A5) in the Appendix. The net power exchanged between these two nanoparticles (radiative heat flux) is

$$
\begin{aligned}
\varphi_{j \leftrightarrow i} & =\varphi_{j \rightarrow i}-\varphi_{i \rightarrow j} \\
& =3 \int_{0}^{+\infty} \frac{d \omega}{2 \pi}\left[\Theta\left(\omega, T_{j}\right)-\Theta\left(\omega, T_{i}\right)\right] \mathcal{T}_{i, j}(\omega),
\end{aligned}
$$

which allows to define the thermal conductance $(G)$ between the particle $i$ and $j$ as $[37,38]$

$$
G_{i j}=\lim _{\delta T \rightarrow 0} \frac{\varphi_{j \leftrightarrow i}}{T_{j}-T_{i}},
$$

where $\delta T=T_{j}-T_{i}$ is the temperature difference between the two particles. Let us now consider finite-size square-lattice nanoparticle ensembles $\mathrm{L}$ and $\mathrm{U}$, as shown in Fig. 1. We can define the radiative heat flux between the two particle ensembles as

$$
\varphi=\sum_{i \in \mathrm{U}} \sum_{j \in \mathrm{L}} \varphi_{j \leftrightarrow i},
$$

where nanoparticle $i$ and $j$ belong to the upper nanoparticle ensemble (U) and the lower nanoparticle ensemble (L), respectively. The total thermal conductance $(G)$ between the ensemble $U$ and ensemble $L$ with a separation is a function of many parameters (e.g., temperature $T$, separation $d$, and lattice spacing $p$, etc.), which is defined as follows:

$$
G(p, d)=\lim _{\delta T \rightarrow 0} \frac{\varphi}{\delta T}=\sum_{i \in \mathrm{U}} \sum_{j \in \mathrm{L}} G_{i j} .
$$

The total thermal conductance $G(p, d)$ between the two ensembles is the sum of the thermal conductances $G_{i j}$ of all possible nanoparticle pairs, where one nanoparticle is from the ensemble $\mathrm{L}$ and the other one is from the ensemble $\mathrm{U}$. The total thermal conductance can also be expressed as the frequency integral of the spectral thermal conductance $G_{\omega}(p, d): G(p, d)=\int_{0}^{+\infty} G_{\omega}(p, d) d \omega$.

\section{Radiative thermal energy emitted by the single nanoparticle ensemble}

The radiative thermal energy at position $\mathbf{r}$ emitted by the particle ensemble can be evaluated by the Poynting vector, 
which is defined as follows $[46,49]$ :

$$
\langle\mathbf{S}(\mathbf{r})\rangle=2 \int_{0}^{+\infty}\langle\mathbf{S}(\mathbf{r}, \omega)\rangle \frac{d \omega}{2 \pi},
$$

where the spectral Poynting vector $\langle\mathbf{S}(\mathbf{r}, \omega)\rangle$ yields

$$
\langle\mathbf{S}(\mathbf{r}, \omega)\rangle=\operatorname{Re}\left\langle\mathbf{E}(\mathbf{r}, \omega) \times \mathbf{H}^{*}(\mathbf{r}, \omega)\right\rangle,
$$

where electric and magnetic field radiated by the fluctuating electric dipole $\left(\mathbf{p}^{f}\right)$ and magnetic dipole $\left(\mathbf{m}^{f}\right)$ yield

$$
\mathbf{E}(\mathbf{r}, \omega)=\mu_{0} \omega^{2} \sum_{i=1}^{N} G^{E E}\left(\mathbf{r}, \mathbf{r}_{i}\right) \mathbf{p}_{i}^{f}+\mu_{0} \omega k \sum_{i=1}^{N} G^{E M}\left(\mathbf{r}, \mathbf{r}_{i}\right) \mathbf{m}_{i}^{f},
$$

$$
\mathbf{H}(\mathbf{r}, \omega)=k \omega \sum_{i=1}^{N} G^{M E}\left(\mathbf{r}, \mathbf{r}_{i}\right) \mathbf{p}_{i}^{f}+k^{2} \sum_{i=1}^{N} G^{M M}\left(\mathbf{r}, \mathbf{r}_{i}\right) \mathbf{m}_{i}^{f},
$$

where $\mu_{0}$ is the vacuum permeability, $N$ is the number of particles. $G^{\nu \tau}\left(\mathbf{r}, \mathbf{r}_{i}\right)(\nu, \tau=E$ or $M)$ is the Green's function connecting the field point $\mathbf{r}$ and the source point $\mathbf{r}_{i}$ in the particle system considering MBI, which is the solution of Eq. (A9) with the help of Eqs. (A8) and (A10) in the Appendix. Substituting Eqs. (9) and (10) into Eq. (8), the spectral Poynting vector can be rearranged as

$$
\begin{aligned}
\langle\mathbf{S}(\mathbf{r}, \omega)\rangle= & \operatorname{Re}\left\langle\mathbf{E}(\mathbf{r}, \omega) \times \mathbf{H}^{*}(\mathbf{r}, \omega)\right\rangle \\
= & \operatorname{Re}\left\{\left\langle\mathbf{x}\left(E_{y} H_{z}^{*}-E_{z} H_{y}^{*}\right)+\mathbf{y}\left(E_{z} H_{x}^{*}-E_{x} H_{z}^{*}\right)\right.\right. \\
& \left.\left.+\mathbf{z}\left(E_{x} H_{y}^{*}-E_{y} H_{x}^{*}\right)\right\rangle\right\} \\
= & \sum_{i=1}^{N} \sum_{n=1}^{3} \sum_{m=1}^{3} \operatorname{Re}\left\{\mu _ { 0 } \omega ^ { 3 } k \left[\mathbf{x}\left(G_{y n}^{E E} G_{z m}^{M E *}-G_{z n}^{E E} G_{y m}^{M E *}\right)\right.\right. \\
& +\mathbf{y}\left(G_{z n}^{E E} G_{x m}^{M E *}-G_{x n}^{E E} G_{z m}^{M E *}\right) \\
& \left.+\mathbf{z}\left(G_{x n}^{E E} G_{y m}^{M E *}-G_{y n}^{E E} G_{x m}^{M E *}\right)\right]\left\langle p_{i, n}^{f} p_{i, m}^{f *}\right\rangle \\
& +\mu_{0} \omega k^{3}\left[\mathbf{x}\left(G_{y n}^{E M} G_{z m}^{M M *}-G_{z n}^{E M} G_{y m}^{M M *}\right)\right. \\
& +\mathbf{y}\left(G_{z n}^{E M} G_{x m}^{M M *}-G_{x n}^{E M} G_{z m}^{M M *}\right) \\
& \left.\left.+\mathbf{z}\left(G_{x n}^{E M} G_{y m}^{M M *}-G_{y n}^{E M} G_{x m}^{M M *}\right)\right]\left\langle m_{i, n}^{f} m_{i, m}^{f *}\right)\right\}, \quad(11)
\end{aligned}
$$

where subscripts $m$ and $n$ are polarization direction index, $\mathbf{x}, \mathbf{y}$, and $\mathbf{z}$ are the unit vectors of $x, y$, and $z$ axes in the given Cartesian coordinate system. $G_{\mu_{1} \mu_{2}}^{\nu \tau}\left[\mu_{1}=x, y, z ; \mu_{2}=\right.$ $(m$ or $n)=1,2,3$ and $v, \tau=E, M]$ is the element of the $3 \times 3$ Green's function $G^{\nu \tau}\left(\mathbf{r}, \mathbf{r}_{i}\right)(\nu, \tau=E$ or $M)$, which is the solution of Eq. (A9) with the help of Eqs. (A8) and (A10) in the Appendix. The fluctuation dissipation theorem yields $[32,33,38]$

$$
\begin{aligned}
\left\langle p_{i, n}^{f} p_{i, m}^{f *}\right\rangle & =2 \frac{\epsilon_{0}}{\omega} \operatorname{Im}\left(\chi_{E}\right) \Theta(\omega, T) \delta_{n m}, \\
\left\langle m_{i, n}^{f} m_{i, m}^{f *}\right\rangle & =\frac{2}{\mu_{0} \omega} \operatorname{Im}\left(\chi_{H}\right) \Theta(\omega, T) \delta_{n m} .
\end{aligned}
$$

Finally, the spectral Poynting vector of Eq. (11) can be rewritten as follows:

$$
\langle\mathbf{S}(\mathbf{r}, \omega)\rangle=2 \sum_{i=1}^{N} \operatorname{Re}\left(k^{3} \mathbb{S} \Theta\left(\omega, T\left(\mathbf{r}_{i}\right)\right)\right),
$$

where $\mathbb{S}$ is defined as follows:

$$
\begin{aligned}
\mathbb{S}= & \sum_{n=m=1}^{3}\left[\mathbf{x}\left(G_{y n}^{E E} G_{z m}^{M E *}-G_{z n}^{E E} G_{y m}^{M E *}\right)\right. \\
& +\mathbf{y}\left(G_{z n}^{E E} G_{x m}^{M E *}-G_{x n}^{E E} G_{z m}^{M E *}\right) \\
& \left.+\mathbf{z}\left(G_{x n}^{E E} G_{y m}^{M E *}-G_{y n}^{E E} G_{x m}^{M E *}\right)\right] \operatorname{Im}\left(\chi_{E}\right) \\
& +\sum_{n=m=1}^{3}\left[\mathbf{x}\left(G_{y n}^{E M} G_{z m}^{M M *}-G_{z n}^{E M} G_{y m}^{M M *}\right)\right. \\
& +\mathbf{y}\left(G_{z n}^{E M} G_{x m}^{M M *}-G_{x n}^{E M} G_{z m}^{M M *}\right) \\
& \left.+\mathbf{z}\left(G_{x n}^{E M} G_{y m}^{M M *}-G_{y n}^{E M} G_{x m}^{M M *}\right)\right] \operatorname{Im}\left(\chi_{H}\right)
\end{aligned}
$$

The Poynting vector emitted by the fluctuating electric and magnetic dipoles can be obtained by Eqs. (14) and (15), which is an extension of the recent work [46] to take the magnetic dipole contribution into consideration and is applicable for not only dielectric, but also metallic nanoparticle ensembles.

\section{Dielectric function and polarizability of nanoparticle}

Three different materials are used in this work, metallic $\mathrm{Ag}$, dielectric $\mathrm{SiC}$, and phase-change $\mathrm{VO}_{2}$, respectively. The dielectric functions of $\mathrm{Ag}$ and $\mathrm{SiC}$ are described by the Drude model $\epsilon(\omega)=1-\omega_{p}^{2} /\left(\omega^{2}+i \gamma \omega\right)$ with parameters $\omega_{p}=1.37 \times 10^{16} \mathrm{rad} \mathrm{s}^{-1}$ and $\gamma=2.732 \times 10^{13} \mathrm{rad} \mathrm{s}^{-1}$ [50] and the Drude-Lorentz model $\epsilon(\omega)=\epsilon_{\infty}\left(\omega^{2}-\omega_{l}^{2}+\right.$ $i \gamma \omega) /\left(\omega^{2}-\omega_{t}^{2}+i \gamma \omega\right)$ with parameters $\epsilon_{\infty}=6.7, \omega_{l}=$ $1.827 \times 10^{14} \mathrm{rad} \mathrm{s}^{-1}, \omega_{t}=1.495 \times 10^{14} \mathrm{rad} \mathrm{s}^{-1}$, and $\gamma=$ $0.9 \times 10^{12} \mathrm{rad} \mathrm{s}^{-1}$ [51], respectively. The $\mathrm{VO}_{2}$ is a kind of phase-change material, which undergoes an insulator-metal transition around $341 \mathrm{~K}$ (phase transition temperature). Below $341 \mathrm{~K}, \mathrm{VO}_{2}$ is a uniaxial anisotropic insulator, of which the dielectric function can be described by a tensor as follows:

$$
\left(\begin{array}{ccc}
\epsilon_{\|} & 0 & 0 \\
0 & \epsilon_{\perp} & 0 \\
0 & 0 & \epsilon_{\perp}
\end{array}\right)
$$

where $\epsilon_{\perp}$ and $\epsilon_{\|}$are ordinary and extraordinary dielectric function component relative to the optic axis of uniaxial insulating $\mathrm{VO}_{2}$, respectively. Both ordinary and extraordinary dielectric function can be described by the Lorentz model as follows:

$$
\epsilon(\omega)=\epsilon_{\infty}+\sum_{k=1}^{N_{L}} \frac{S_{k} \omega_{k}^{2}}{\omega_{k}^{2}-i \gamma_{k} \omega-\omega^{2}},
$$

where $S_{k}, \omega_{k}$, and $\gamma_{k}$ are phonon strength, phonon frequency, and damping coefficient of the $k$ th phonon mode. $N_{L}$ is the number of phonon modes. All the necessary parameters for both $\epsilon_{\perp}$ and $\epsilon_{\|}$can be found in the Ref. [52]. Above $341 \mathrm{~K}$, $\mathrm{VO}_{2}$ is an isotropic metal, of which the dielectric function can be described by a Drude model as follows [52]:

$$
\epsilon(\omega)=\epsilon_{\infty} \frac{\omega_{p}^{2}}{\omega^{2}-i \omega \gamma}
$$

where $\epsilon_{\infty}=9, \omega_{p}=1.51 \times 10^{15} \mathrm{rad} \mathrm{s}^{-1}$, and $\gamma=1.88 \times$ $10^{15} \mathrm{rad} \mathrm{s}^{-1}$. 
TABLE I. Asymptotic regimes of RHT between 2D finite-size square-lattice nanoparticle ensembles.

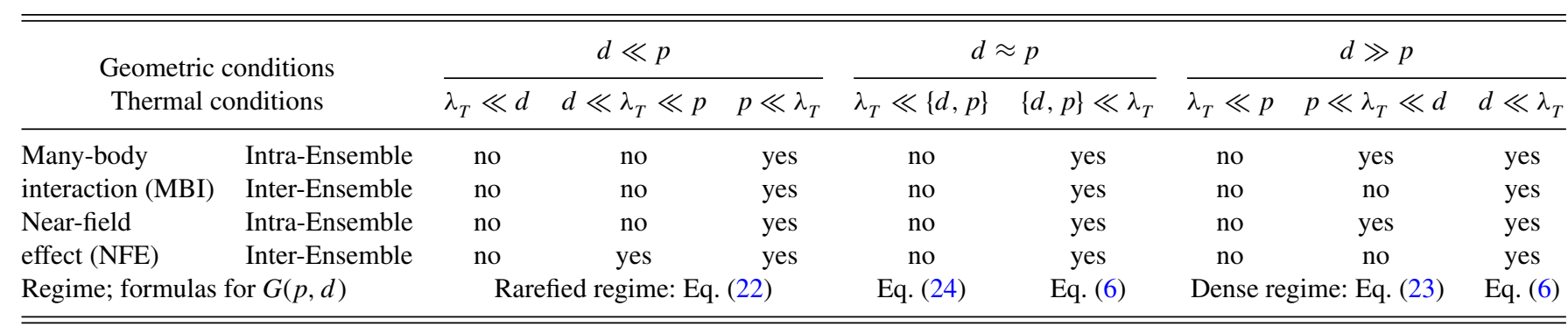

For a nanoparticle composed of an isotropic material (e.g., metallic $\mathrm{Ag}$ and dielectric $\mathrm{SiC}$ ), the electric and magnetic dipole polarizabilities are given as follows [9]:

$$
\alpha_{E}=4 \pi a^{3} \frac{\varepsilon-1}{\varepsilon+2}
$$

where $\varepsilon$ is the relative permittivity and

$$
\alpha_{H}=\frac{2 \pi}{15} a^{3}\left(\frac{\omega a}{c}\right)^{2}(\epsilon-1)
$$

The polarizability of $\mathrm{Ag}$ and $\mathrm{SiC}$ nanoparticle can be found in our previous work $[33,38,40]$.

However, for the anisotropic insulator-phase $\mathrm{VO}_{2}$ nanoparticle below the transition temperature, a well-established solution is applied to the polarizability of anisotropic spherical nanoparticle in two steps: first calculate polarizability for nanoparticle using $\epsilon_{\perp}$ and $\epsilon_{\|}$separately, and then add up the results according to the $1 \backslash 3-2 \backslash 3$ rule [53]:

$$
\alpha_{\nu}=\frac{2}{3} \alpha_{v}\left(\epsilon_{\perp}\right)+\frac{1}{3} \alpha_{v}\left(\epsilon_{\|}\right),
$$

where $v=E$ or $H$. The electric and magnetic polarizabilities for both insulator-phase and metallic-phase $\mathrm{VO}_{2}$ nanoparticles are shown in Fig. 3. In order to compare the resonance frequency to the characteristic thermal frequency, the spectral radiance of the blackbody at room temperature is also added in Fig. 3(b) for reference. There is a mismatch between the characteristic thermal frequency and the polarizability resonance frequency of metallic $\mathrm{VO}_{2}$ nanoparticle. A similar mismatch between characteristic thermal frequency and polarizability resonance frequency of $\mathrm{Ag}$ nanoparticle as that of metallic $\mathrm{VO}_{2}$ nanoparticle can also be observed [40].

\section{RADIATIVE HEAT TRANSFER BETWEEN 2D FINITE-SIZE PERIODIC SQUARE-LATTICE NANOPARTICLE ENSEMBLES: ASYMPTOTIC REGIMES}

In this section we discuss the main asymptotic regimes of the RHT between 2D periodic finite-size square-lattice nanoparticle ensembles. In general, we have four length scales in this problem. Three of them are geometric length scales: the lattice spacing $p$, the separation $d$ between the 2D systems, and the nanoparticle radius $a$. The fourth is a thermal length scale: the characteristic thermal wavelength $\lambda_{T}$. In this work, we keep fixed the value of $a=20 \mathrm{~nm}$, and set all the three other length scales much larger than $a$. We will see (Sec. IV B) that a fifth additional length scale, related to emergence of multiple scattering of the electromagnetic between the two nanoparticles of a pair, will naturally emerge in this problem.
According to combinations of the geometric length scales $\{p, d\}$, three kinds of 2D finite-size square-lattice nanoparticle ensembles can be clarified, which are shown in Fig. 4.

The MBI effects and near-field effects (NFE) on RHT are significantly dependent on the combination of $\left\{p, d, \lambda_{T}\right\}$, and different asymptotic regimes can be identified, as listed in the

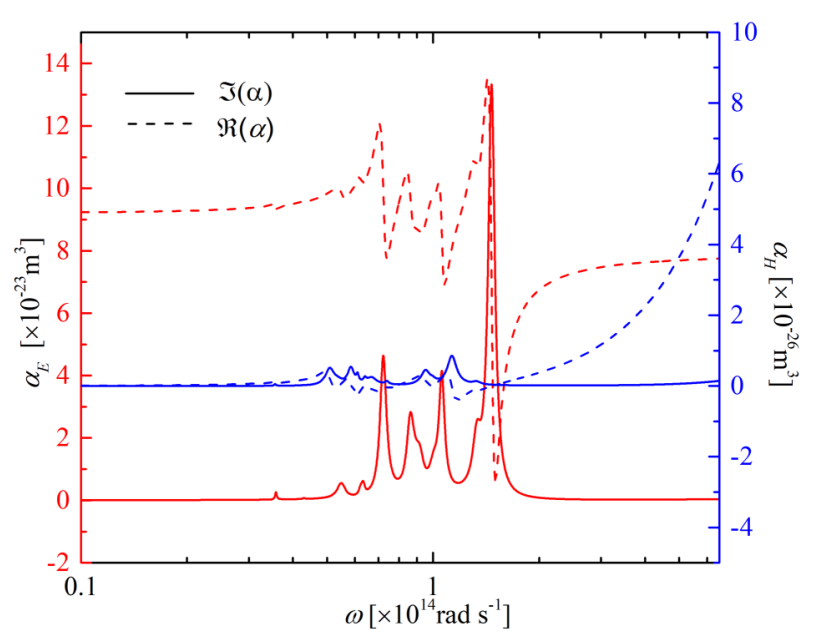

(a) Insulator $\mathrm{VO}_{2}$

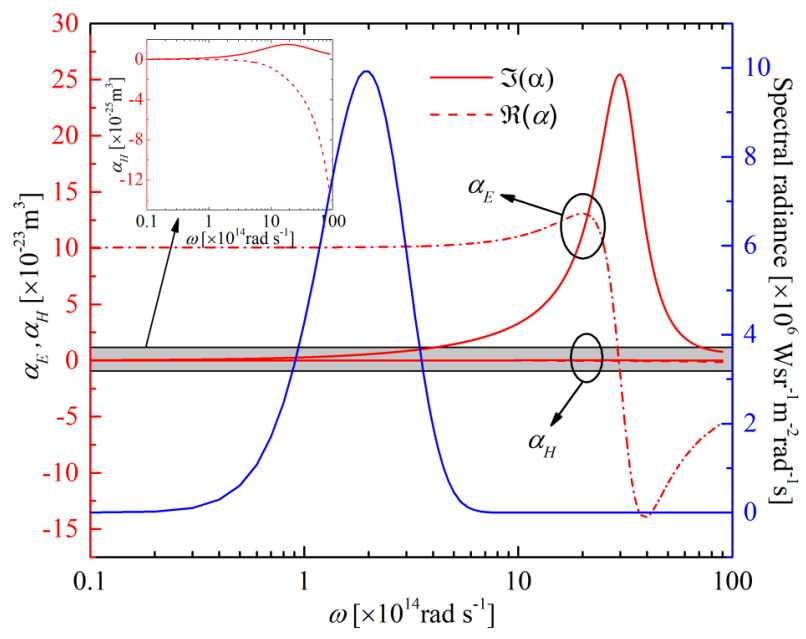

(b) metallic $\mathrm{VO}_{2}$

FIG. 3. The electric and magnetic polarizabilities for both the (a) insulator-phase $\mathrm{VO}_{2}$ nanoparticle and (b) metallic-phase $\mathrm{VO}_{2}$ nanoparticle. Nanoparticle radius $a$ is $20 \mathrm{~nm}$. For insulator $\mathrm{VO}_{2}$ particle, the " $1 \backslash 3-2 \backslash 3$ " rule is applied to calculate the polarizability with the help of $\epsilon_{\|}$and $\epsilon_{\perp}$ [53]. The spectral radiance of the blackbody at room temperature is also added for reference. 


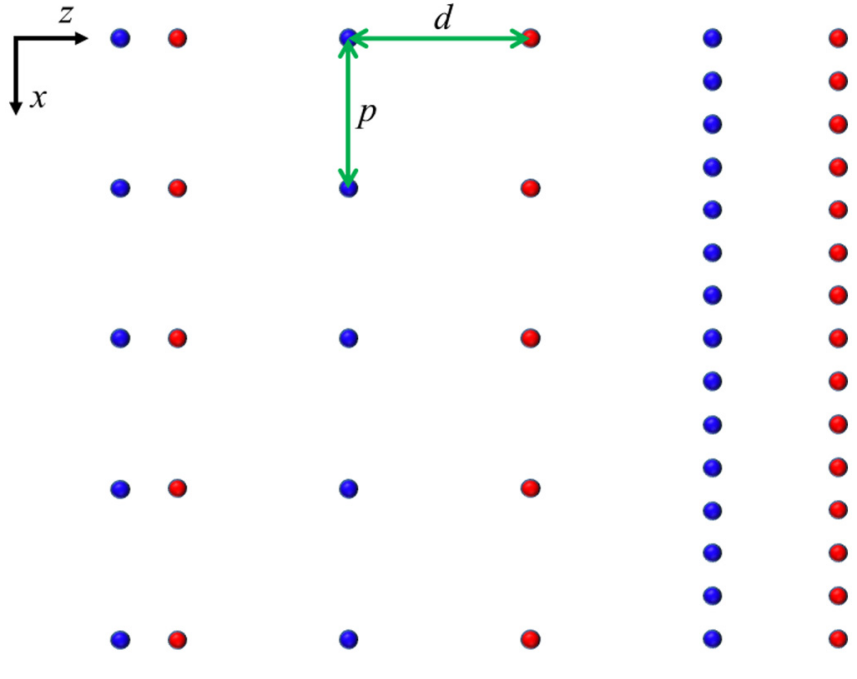

(a) (b)

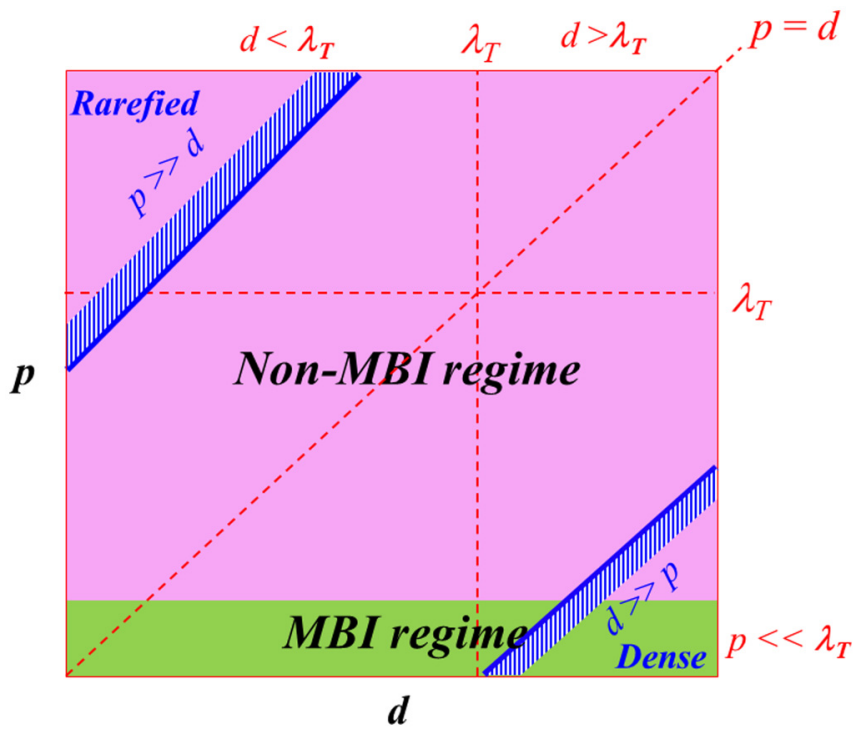

FIG. 5. Asymptotic regime map of RHT between 2D finite-size square-lattice nanoparticle ensembles. Combined with Table I, four asymptotic regimes are identified, MBI regime, non-MBI regime, rarefied regime, and dense regime, respectively.

between the nanoparticle ensembles in this regime can be simplified as follows:

$$
G(p, d)=N \times G_{2 n p}(d)
$$

where $G(p, d)$ is the radiative thermal conductance between the two nanoparticle ensembles with the separation $d$ and lattice spacing $p, N$ is the number of nanoparticles in each ensemble, $G_{2 n p}(d)$ is the thermal conductance between two isolated nanoparticles with the separation $d$, which can be calculated easily by Eq. (4). The thermal conductance between ensembles composed of a lot of nanoparticles can be easily obtained by the simplified equation (22) rather than by the general formula (6).

\section{B. Dense regime}

In this regime, each ensemble behaves like a solid-dense body when considering heat exchange between two 2D nanoparticle ensembles. Nanoparticles in each ensemble are indistinguishable and have nearly the same contribution to thermal radiation with each other. In this regime, the general formula (6) for the thermal conductance $G(p, d)$ between two nanoparticle ensembles can be simplified as follows:

$$
G(p, d)=N^{2} \times G_{2 n p}(d) .
$$

As compared to Eq. (22) used for the rarefied regime, in the dense regime thermal conductance between two finite-size square-lattice nanoparticle ensembles in Eq. (23) is $N^{2} \times$ $G_{2 n p}(d)$ rather than $N \times G_{2 n p}(d)$. The thermal conductance between ensembles composed of a lot of nanoparticles can be easily obtained by the simplified equation (23) of the general formula (6). 


\section{Non-MBI regime}

In this regime, many-body interaction effects on RHT are negligible. Hence, the thermal conductance $G(p, d)$ between two ensembles is the simple pairwise sum of the thermal conductance for all possible $N^{2}$ pairs and, being purely additive, it neglects all possible MBI effects. Indeed, $G(p, d)$ is defined as

$$
G(p, d)=\sum_{\text {pairwise }}^{N^{2}} G_{\text {pair }}
$$

where $G_{\text {pair }}$ is the thermal conductance between the two particles of an isolated pair (one particle in $\mathrm{L}$ and the another in $\mathrm{U}$ ) calculated using Eq. (4) and assuming the pair as completely isolated (i.e., all the other $2 N-2$ particles are absent).

\section{MBI regime}

In this regime, due to the complex many-body interaction, the simplified equation (24) of the general formula (6) for the thermal conductance in the non-MBI regime cannot be used anymore. The thermal conductance must be calculated using Eq. (6) with the help of the exact transmission coefficient of Eq. (2) since no approximation is possible.

Until now, we have clearly identified four asymptotic regimes of RHT between 2D finite-size square-lattice nanoparticle ensembles in total: rarefied regime, dense regime, non-MBI regime, and MBI regime, respectively. As can been seen in Fig. 5 and Table I, the MBI regime covers a part of the dense regime and the rest part of the dense regime is covered by the non-MBI regime. Figure 5 combined with Table I can be easily applied to determine the regime of RHT between 2D finite-size square-lattice nanoparticle ensembles.

In columns $1,2,4$, and 6 , there is no MBI effect corresponding to the non-MBI regime and the thermal conductance can be calculated directly by the simplified equation (24) rather than the general formula (6) treating all possible nanoparticle pairs as they were isolated in vacuum without any influence by other nanoparticles. The simple equation (22) can be easily applied to obtain the thermal conductance under such conditions listed in the columns 1,2, and 3 corresponding to the rarefied regime. Columns 1 and 2 belong to both rarefield regime and non-MBI regime, therefore, simplification of the calculation for the thermal conductance can go further by using Eq. (22) as compared to that by Eq. (24) simplified from the general formula (6). Condition in the column 6 belongs to both non-MBI regime and dense regime, where the efficient Eq. (23) can be applied to simplify the calculation of the thermal conductance as compared to the simplified equation (24) for the non-MBI regime.

It is also worthwhile to mention that there are some other length scales that might influence the thermal behavior.

(a) The size of the ensemble. When the size of the ensemble is large enough, the boundary effect on radiative heat transfer is negligible and finite ensemble of such large size starts mimic the infinite ensemble. The effect of the size of ensembles will be discussed at the end of the following Sec. IV A in brief.

(b) The resonance wavelength of the nanoparticles. (c) The length at which the contribution of the electric dipoles and magnetic dipoles to the radiative heat transfer is comparable. Since the inhibition and enhancement of the electric dipole contribution and magnetic contribution to NFRHT might occur at different length scales. The total thermal conductance is the result of the competition between the electric and magnetic dipoles.

\section{RADIATIVE HEAT TRANSFER BETWEEN 2D FINITE-SIZE SQUARE-LATTICE NANOPARTICLE ENSEMBLES: NUMERICAL RESULTS}

In this section, we numerically investigate the RHT between 2D finite-size square-lattice nanoparticle ensembles. Several materials have been considered, e.g., metallic Ag, dielectric $\mathrm{SiC}$, and phase-change material $\mathrm{VO}_{2}$. Regimes of RHT between 2D finite-size square-lattice nanoparticle ensembles under given conditions are identified numerically. Effect of the many-body interaction on the RHT between 2D finite-size square-lattice nanoparticle ensembles is analyzed as focus. In addition, effects of the phase change of material and lateral translation of the two parallel 2D ensembles on RHT are analyzed. Particle radius $(a)$ is $20 \mathrm{~nm}$. The separation distance between any two particles center to center in the particle ensemble is larger than $3 a$, which makes the dipole approximation valid $[32,33,46]$.

\section{A. Regime of RHT between 2D finite-size square-lattice nanoparticle ensembles}

First of all, in order to investigate the regime of RHT between 2D finite-size square-lattice nanoparticle ensembles, we give a general description of the dependence of the scaled thermal conductance $[G(p, d) / N]$ on the parameters $p$ and $d$, as shown in Fig. 6 . Here we define the parameter $\Delta=$ $\frac{p}{d}$, and we set $T=300 \mathrm{~K}, N=400$, and $p=60 \mathrm{~nm}, 160$ $\mathrm{nm}, 320 \mathrm{~nm}, 500 \mathrm{~nm}, 3 \mu \mathrm{m}, 7 \mu \mathrm{m}, 10 \mu \mathrm{m}$, and $20 \mu \mathrm{m}$, respectively. Lines corresponding to $d=0.44 \mu \mathrm{m}, d=\lambda_{T}$, $d=20.04 \mu \mathrm{m}$, and power law $\left(\sim d^{-2}\right.$ and $\left.\sim d^{-6}\right)$ are added for reference. $G(p, d) / N \sim d$ calculated by Eqs. (22) and (23) are also added for reference. The thermal conductance $G(p, d) / N$ corresponding to $\Delta=1(d=p)$ is also added for reference.

As shown in Fig. 6, when $d \ll \lambda_{T}$ and $\Delta \gg 1(p \gg d)$, the thermal conductance $G(p, d) / N$ follows the same power law $d^{-6}$ as that calculated by Eq. (22). When $d \gg \lambda_{T}$ and $\Delta \ll 1(p \ll d)$, the thermal conductance $G(p, d) / N$ follows the same power law $d^{-2}$ as that calculated by Eq. (23). For a fixed separation $d$, the thermal conductance increases with the decreasing lattice spacing $p$. The nanoparticles inside each ensemble move from the far field to the near field of its nearby nanoparticles when $p$ decreases from $20 \mu \mathrm{m}$ to 60 $\mathrm{nm}$. The near-field effect accounts for the increasing thermal conductance with decreasing $p$. As can be seen in Fig. 6, Eqs. (22) and (23) give the lower and upper limits for the thermal conductance, respectively.

Then, based on the general description of the thermal conductance as shown in Fig. 6, in order to numerically figure out the regime of RHT, a new parameter $\psi(p, d)$ is defined as 


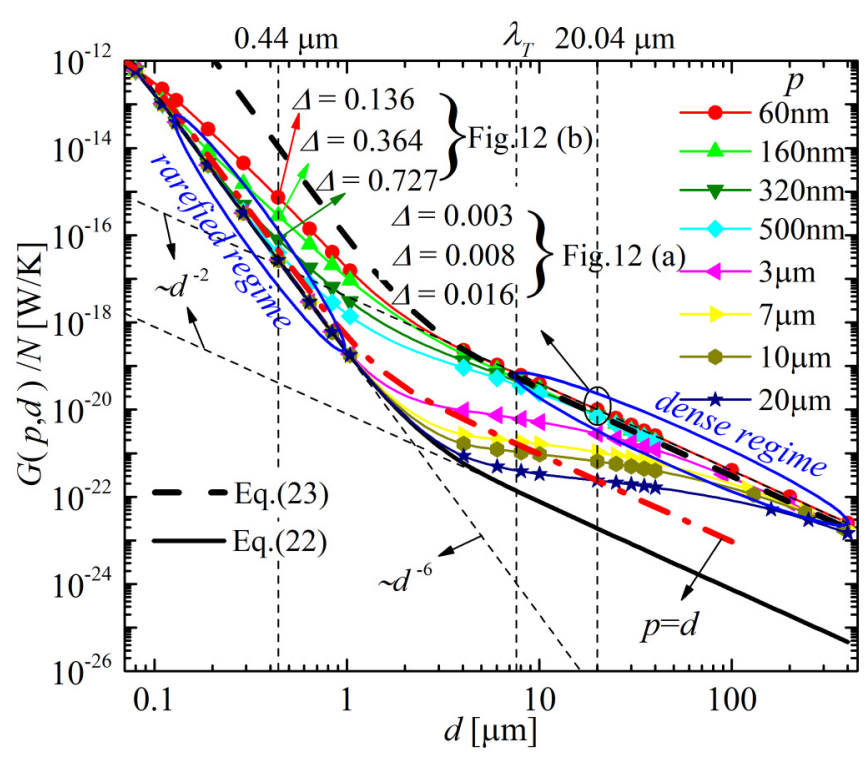

FIG. 6. The scaled thermal conductance $[G(p, d) / N]$ between 2D finite-size square-lattice nanoparticle ensembles as a function of separation distance $d$. We define $\Delta=\frac{p}{d}$, and set $T=300 \mathrm{~K}$, $a=20 \mathrm{~nm}, N=400$, and $p=60 \mathrm{~nm}, 160 \mathrm{~nm}, 320 \mathrm{~nm}, 500 \mathrm{~nm}$, $3 \mu \mathrm{m}, 7 \mu \mathrm{m}, 10 \mu \mathrm{m}$, and $20 \mu \mathrm{m}$, respectively. Fitting lines of $d=0.44 \mu \mathrm{m}, d=\lambda_{T}, d=20.04 \mu \mathrm{m}$, and power law $\left(\sim d^{-2}\right.$ and $\sim d^{-6}$ ) are added for reference. $G(p, d) / N$ calculated by Eqs. (22) and (23) as functions of $d$ are also added for reference. The thermal conductance $G(p, d) / N$ corresponding to $\Delta=1(d=p)$ is also added for reference. The data points used in the analysis on the thermal conductance spectrum in Fig. 12 are marked for reference. Two regimes (rarefied regime and dense regime) are circled out in blue.

follows:

$$
\psi(p, d)=\frac{G(p, d)}{G_{S}(p, d)},
$$

where $G(p, d)$ is the thermal conductance calculated by the general formula (6), $G_{S}(p, d)$ is the thermal conductance calculated by the simplified formulas of the general formula (6), i.e., Eqs. (22), (23), and (24). The data points corresponding to the rarefied regime and dense regime have been circled out in blue in Fig. 6.

\section{Rarefied regime}

In order to identify the rarefied regime, the parameter $\psi(p, d)$ defined by Eq. (25) is applied, where $G_{S}(p, d)$ is calculated by the simplified equation (22). We calculate the thermal conductance $G_{S}(p, d)$ in the considered domain $(p, d)$ and give the contour of $\psi(p, d)$ in Fig. 7. In the contour, the rarefied regime is corresponding to the region, where $\psi(p, d) \approx 1$. Under conditions that $T=300 \mathrm{~K}$ and $a=20$ $\mathrm{nm}$, the region corresponding to the rarefied regime is clearly identified in Fig. 7. In this region, the thermal conductance can be easily calculated by the simplified equation (22) of the general formula (6).

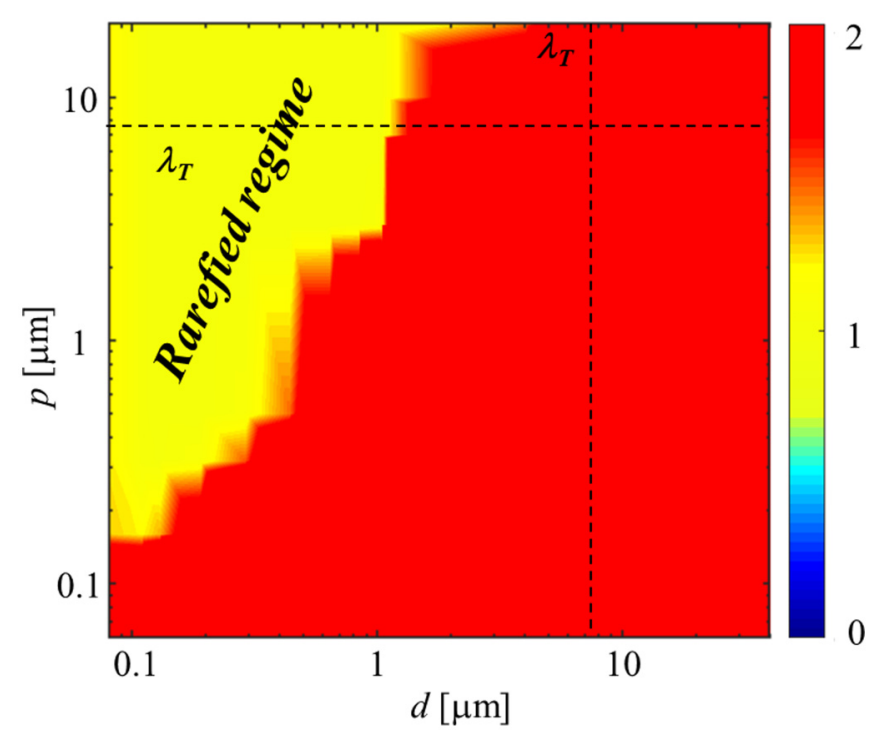

FIG. 7. Contour of $\psi(p, d)$ calculated with the help of Eq. (22). The rarefied regime is corresponding to the domain, where $\psi(p, d) \approx 1 . T=300 \mathrm{~K}, a=20 \mathrm{~nm} . N=400$.

\section{Dense regime}

In order to identify the dense regime clearly, the thermal conductance $G_{S}(p, d)$ calculated by Eq. (23) is applied to calculate $\psi(p, d)$ in the considered domain $(p, d)$ and the contour of $\psi(p, d)$ is shown in Fig. 8. In the contour, the dense regime is corresponding to the region, where $\psi(p, d) \approx 1$. In this region, the thermal conductance between two ensembles composed of many nanoparticles can be easily calculated by the simplified equation (23) of the general formula (6).

In addition to the numerical identification for the rarefied regime and dense regime under the considered parameters for the ensembles, the effect of size of lattice on the RHT is also analyzed in both near field and far field. The spectral thermal

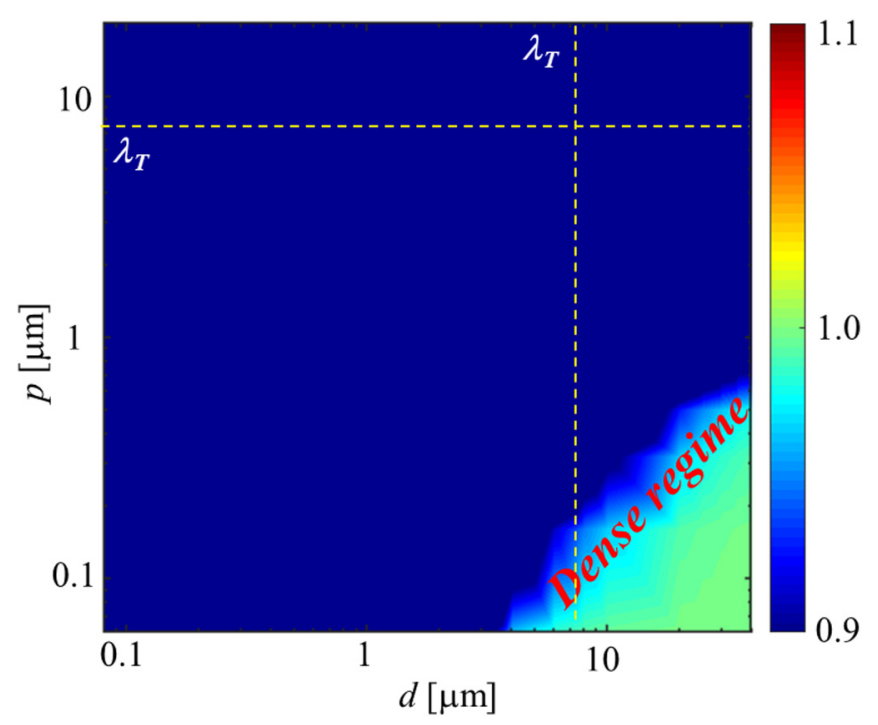

FIG. 8. Contour of $\psi(p, d)$ calculated with the help of Eq. (23). The dense regime is corresponding to the domain, where $\psi(p, d) \approx$ 1. $T=300 \mathrm{~K}, a=20 \mathrm{~nm}$. $N=400$. 


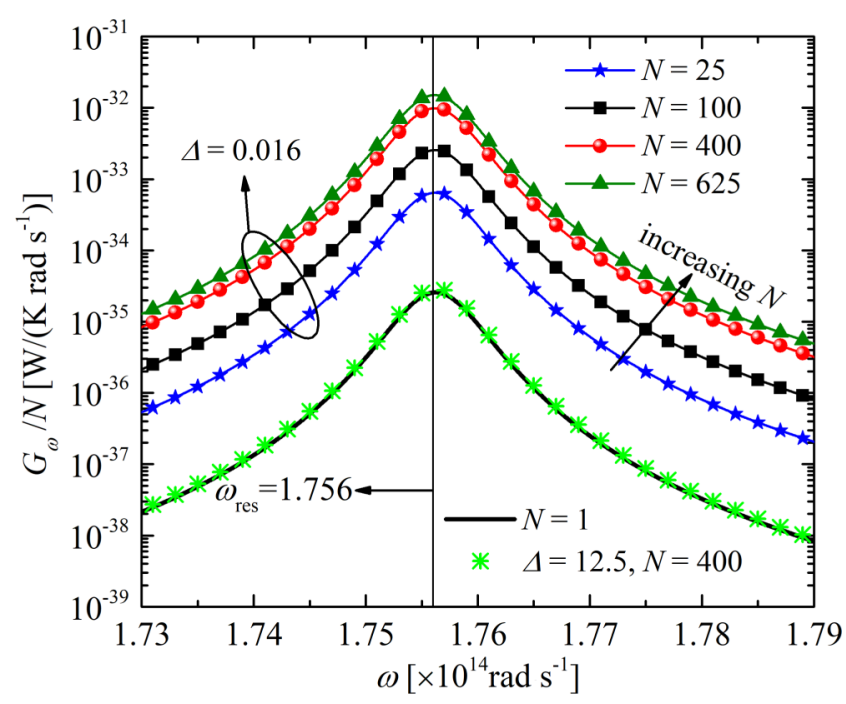

(a) Separation $d=20 \mu \mathrm{m}+2 a$ for the far-field case

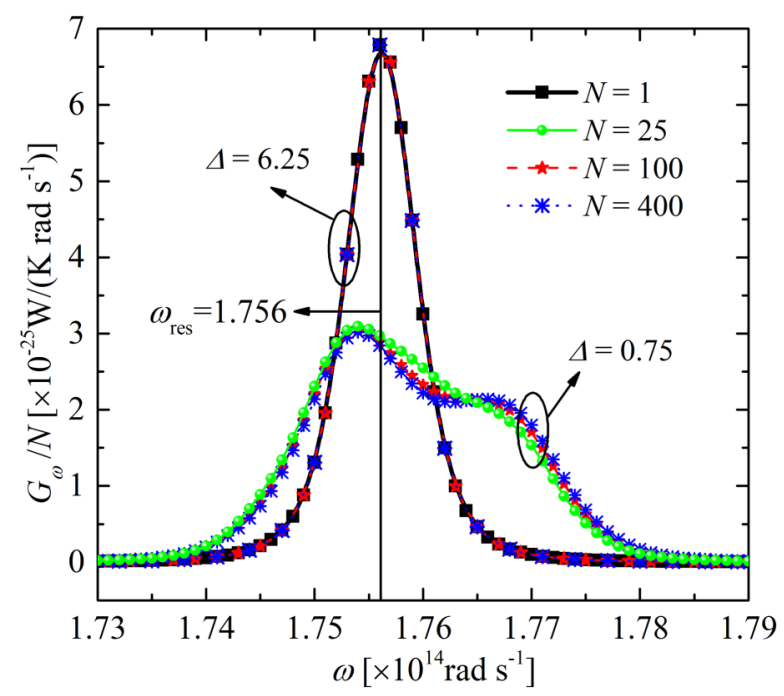

(b) Separation $d=40 \mathrm{~nm}+2 a$ for the near-field case

FIG. 9. The spectral thermal conductance $G_{\omega}$ between SiC 2D finite-size square-lattice nanoparticle ensembles is scaled by the number of nanoparticles in each ensemble $N$. Two cases are considered: (a) separation $d=20 \mu \mathrm{m}+2 a$ between the two ensembles center to center for the far-field case, two cases have been considered, $\Delta=0.016$ and 12.5 , respectively. and (b) separation $d=40 \mathrm{~nm}$ $+2 a$ for the near-field case, $\Delta=0.75$ and 6.25. The thermal conductance spectrum between two nanoparticles separated by the same separation $d$ as that of the 2D ensemble is also added, which is the case $N=1$ in (a) and (b). The polarizability resonance frequency of the single $\mathrm{SiC}$ nanoparticle is added for reference, shown as $\omega_{\text {res }}=1.756 \times 10^{14} \mathrm{rad} \mathrm{s}^{-1}$.

conductance between two $\mathrm{SiC} 2 \mathrm{D}$ finite-size square-lattice nanoparticle ensembles separated by two different distances $d$ scaled by the amount of nanoparticles in each ensemble $N$ is shown in Figs. 9(a) and 9(b). The separation between the two 2D nanoparticle ensembles center to center are $d=20$ $\mu \mathrm{m}+2 a$ (far-field case) and $40 \mathrm{~nm}+2 a$ (near-field case), respectively. The thermal conductance spectrum between two isolated nanoparticles is also added for reference. The amount of nanoparticles in the 2D ensemble varies from case to case.
The thermal conductance spectrum converges with increasing the number of nanoparticles in the 2D ensemble. An ensemble composed of 400 nanoparticles is sufficient to mimic the infinite 2D nanoparticle ensemble when considering heat exchange between the 2D ensembles. As can be seen in Figs. 9(a) and 9(b), thermal conductance $G_{\omega}(p, d) / N$ reduces to that of two isolated nanoparticles when $\Delta \gg 1(p \gg d)$ in both near field and far field, which is corresponding to the rarefied regime.

\section{B. Effect of many-body interaction on RHT between 2D finite-size square-lattice nanoparticle ensembles}

Due to multiple scattering in the ensemble composed of many particles, the many-body interaction has complex effect on RHT. Previous work focused on many-body interaction in particle system with different spatial arrangement (threenanoparticle system [32,54,55], 1D chain of nanoparticles [40], 2D fractal nanoparticle ensemble [48], clusters composed of hundreds of nanoparticles [37,38], and nanoparticles with a substrate $[14,15]$ ), which can not only enhance but also can inhibit NFRHT, and even have negligible effect on NFRHT. Here in this section, we focused on 2D squarelattice nanoparticle ensembles. Both quantitative and qualitative analyses on the many-body effect on NFRHT between 2D finite-size square-lattice nanoparticle ensemble are conducted.

The $\psi(p, d)$ defined by Eq. (25) is applied to evaluate the many-body interaction quantitatively, where $G(p, d)$ is the thermal conductance between nanoparticle ensembles with the many-body interaction calculated by the general formula (6) and $G_{S}(p, d)$ is the thermal conductance between nanoparticle ensembles without the many-body interaction calculated by the simplified pairwise summation formula (24). Here, the ratio $\psi(p, d)$ reflects the presence of MBI effects, and its numerical evaluation is shown in Fig. 10 (the parameters are listed in the caption of the figure). When $\psi(p, d) \approx 1$ (yellow region), the MBI is negligible. When $\psi(p, d)<1$ (blue region), the MBI inhibits the RHT. When $\psi(p, d)>1$ (red region), the MBI enhances the RHT. The oblique blue-dashed line corresponds to $p=d$. Lines for $p=\lambda_{T}$ and $d=\lambda_{T}$ are added. The two red-dashed lines delimitate the rarefied regime and dense regime regions, already discussed in Sec. IV A.

By looking at the value of $\psi(p, d)$, two main regions can be identified, and separated with a black-bold-dashed horizontal line $p \approx 150 \mathrm{~nm}$ in Fig. 10: (1) the non-MBI regime (yellow region) corresponding to $\psi(p, d) \approx 1$ is essentially above that line, and (2) the MBI regime (blue/red regions) corresponding to $\psi(p, d) \neq 1$ is below that line. The MBI regime only occupies a small part of the whole domain, where $p \ll \lambda_{T}$. The rest large domain is corresponding to the non-MBI regime, where MBI effects on RHT can be neglected safely and Eq. (24) is an excellent, time-saving, approximation of Eq. (6). If we focus on the MBI regime $\left(p \ll \lambda_{T}\right)$, we see that for $d<\lambda_{T}$ the MBI significantly inhibits the RHT, and $\psi(p, d)$ reaches the minimum value $\psi(p, d) \approx 0.4$. For the region $d>\lambda_{T}$, on the contrary, MBI enhances the RHT, and $\psi(p, d)$ reaches its maximum value $\psi(p, d) \approx 1$.4. This enhancement effect is modest if compared to what happens between two nanoparticles due to the insertion of a third nanoparticle [32]. 


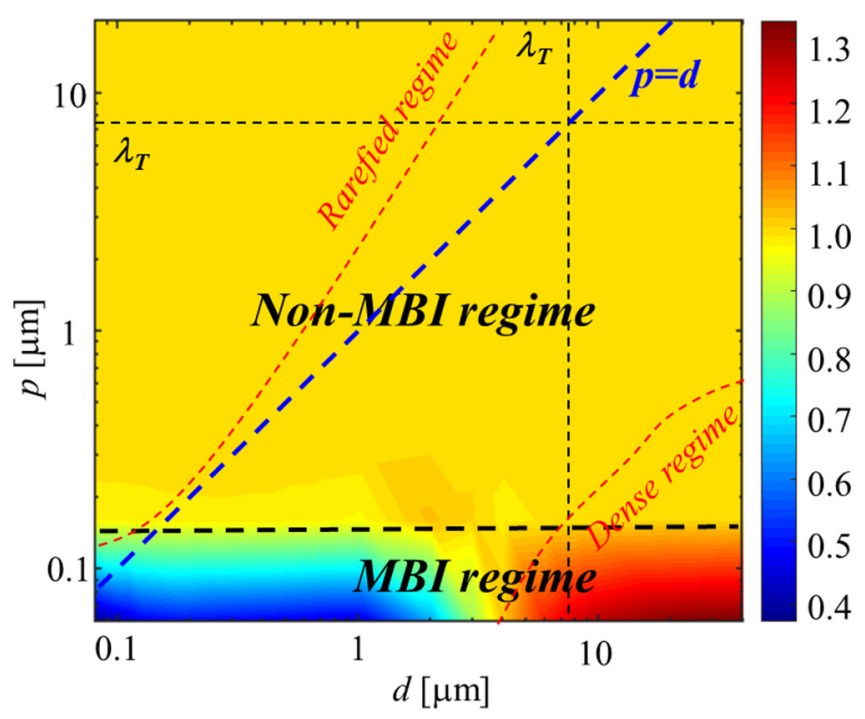

FIG. 10. Contour of the ratio $\psi(p, d)$ for 2D SiC finite-size square-lattice nanoparticle ensembles as a function of $d$ and $p$. The black-bold-dashed lines are the borderlines for the MBI regime. The oblique blue-dashed line corresponds to $p=d$. Lines for $p=\lambda_{T}$ and $d=\lambda_{T}$ are added. $a=20 \mathrm{~nm}, T=300300 \mathrm{~K}, N=400$. The two red-dashed lines delimitate the rarefied regime and dense regime regions, already discussed in Sec. IV A.

In the MBI sector there is a transition region around $d=\lambda_{T}$, where $\psi(p, d) \approx 1$.

In Fig. 10, the horizontal line distinguishing the MBI regime from the non-MBI regime is at $p \approx 150 \mathrm{~nm}$. We try now to understand the origin of this length scale. Let us start by investigating the multiple scattering occurring between the two nanoparticles of a single isolated pair. To this purpose, in Fig. 11 we show the ratio of $G_{2 n p}$ to $G_{0,2 n p}$ as a function of the separation $h$, where $G_{2 n p}$ is the complete thermal conductance between the two nanoparticles calculated using Eq. (4), while $G_{0,2 n p}$ is the thermal conductance neglecting the multiple scattering between the nanoparticles. The expression for $G_{0,2 n p}$ can be easily obtained from Eqs. (3) and (4) with the help of the transmission coefficient in vacuum [see Eq. (30) of [38]], which yields

$$
\begin{aligned}
\mathcal{T}_{i, j}^{0}(\omega)= & \frac{4}{3} k^{4}\left[\operatorname{Im}\left(\chi_{E}^{i}\right) \operatorname{Im}\left(\chi_{E}^{j}\right) \operatorname{Tr}\left(G_{0, i j}^{E E} G_{0, i j}^{E E \dagger}\right)\right. \\
& +\operatorname{Im}\left(\chi_{E}^{i}\right) \operatorname{Im}\left(\chi_{H}^{j}\right) \operatorname{Tr}\left(G_{0, i j}^{E M} G_{0, i j}^{E M \dagger}\right) \\
& +\operatorname{Im}\left(\chi_{H}^{i}\right) \operatorname{Im}\left(\chi_{E}^{j}\right) \operatorname{Tr}\left(G_{0, i j}^{M E} G_{0, i j}^{M E \dagger}\right) \\
& \left.+\operatorname{Im}\left(\chi_{H}^{i}\right) \operatorname{Im}\left(\chi_{H}^{j}\right) \operatorname{Tr}\left(G_{0, i j}^{M M} G_{0, i j}^{M M \dagger}\right)\right],
\end{aligned}
$$

where $G_{0, i j}^{\nu \tau}(\nu, \tau=E$ or $M)$ is the Green function in the free space, of which the explicit expression is given in Eqs. (A1)(A3). In Fig. 11 the ratio $G_{2 n p} / G_{0,2 n p}$ calculated for $\mathrm{SiC}$ nanoparticles (radius $a=20 \mathrm{~nm}, T=300 \mathrm{~K}$ ) shows that scattering effects are relevant only for separations $h<L$, where $L \approx 150 \mathrm{~nm}$ is now a new length scale. When $L<h<$ $\lambda_{T} \approx 7 \mu \mathrm{m}$, the system is in the near field, while the multiple scattering is not relevant. We also note that the interaction between the two nanoparticles always inhibits the thermal conductance. We hence observe that the length scale $L$, setting the occurrence of multiple scattering inside an isolated pair, is

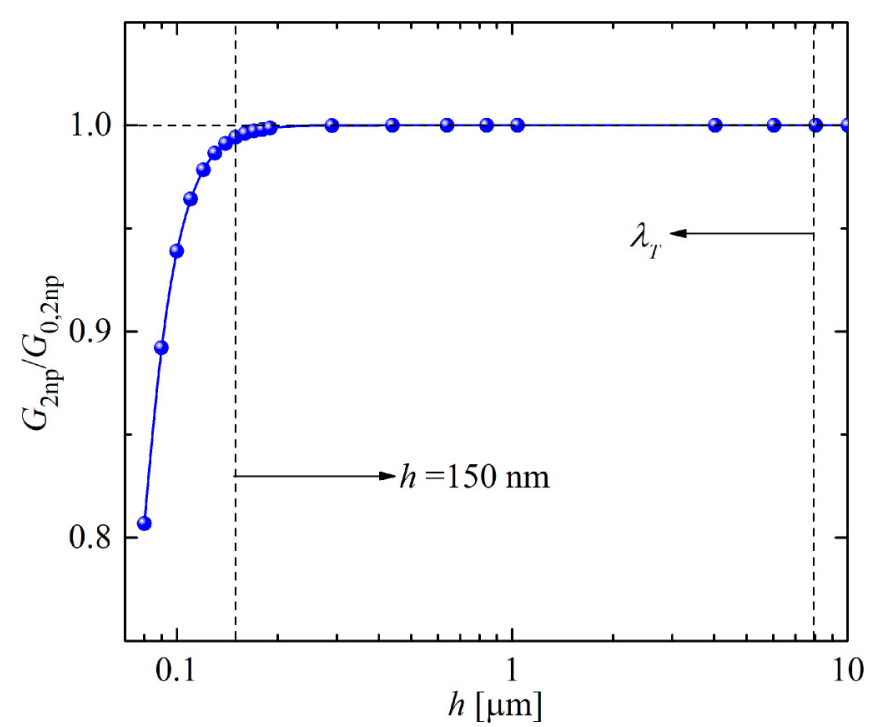

FIG. 11. The ratio of the thermal conductance between two SiC nanoparticles with considering interaction between the two nanoparticles $G_{2 n p}$ by Eq. (4) to that without interaction between the two nanoparticles $G_{0,2 n p}$ as a function of separation $h . a=20 \mathrm{~nm}, T=$ $300 \mathrm{~K}$. The lines corresponding to $h=150 \mathrm{~nm}$ and $h=\lambda_{T}$ are added for reference.

compatible with the value $p \approx 150 \mathrm{~nm}$ setting the transition between non-MBI and MBI regions in Fig. 10. From the same figure, we see that $L$ only affects the lattice spacing $p$, while has no signatures on the separation $d$ between the 2D planes. Hence, we deduce that the necessary condition to have MBI is the multiple scattering between the particles of the same plane. We also stress that the (less restrictive) near-field condition between in-plane particles $\left(p<\lambda_{T}\right)$ is not sufficient to have MBI, and a multiple scattering (i.e., $p<L$ ) is needed. Provided $p<L$, the MBI can also be present when the two 2D systems are in the far field $\left(d>\lambda_{T}\right)$. It is also remarkable that if $d<L$ but $\lambda_{T}>p>L$, we have multiple scattering between the particles of the opposite planes, but we do not have MBI.

In order to better understand the different enhancing and inhibiting MBI effects observed in Fig. 10, we consider below two cases for which we specifically analyze the spectral thermal conductance $G_{\omega}(p, d)$ as shown in Fig. 12: (a) $d>$ $\lambda_{T}(d=20 \mu \mathrm{m}+2 a$, MBI effect enhances RHT) and (b) $d<$ $\lambda_{T}(d=400 \mathrm{~nm}+2 a$, MBI effect inhibits RHT $)$.

\section{Case 1: MBI enhances the RHT $\left\{p \ll \lambda_{T}\right\} \cap\left\{d>\lambda_{T}\right\}$}

In this case, corresponding to the red region of Fig. 10, the MBI effect enhances the RHT. When two ensembles are separated by a large distance (far-field case, e.g., $d=20$ $\left.\mu \mathrm{m}+2 a>\lambda_{T}\right)$, the thermal conductance spectrum has only one peak as can be seen in Fig. 12(a) and the ratio $\psi(p, d)$ is near to but larger than 1 . The maximum $\psi(p, d)$ in this case approximately equals to 1.4 . The value of the thermal conductance is less affected due to the weak intra-ensemble MBI effects inside each ensemble in this case 1 . The frequency corresponding with the peak of the thermal conductance spectrum shows a blueshift behavior with the increasing $\Delta$. In addition, 


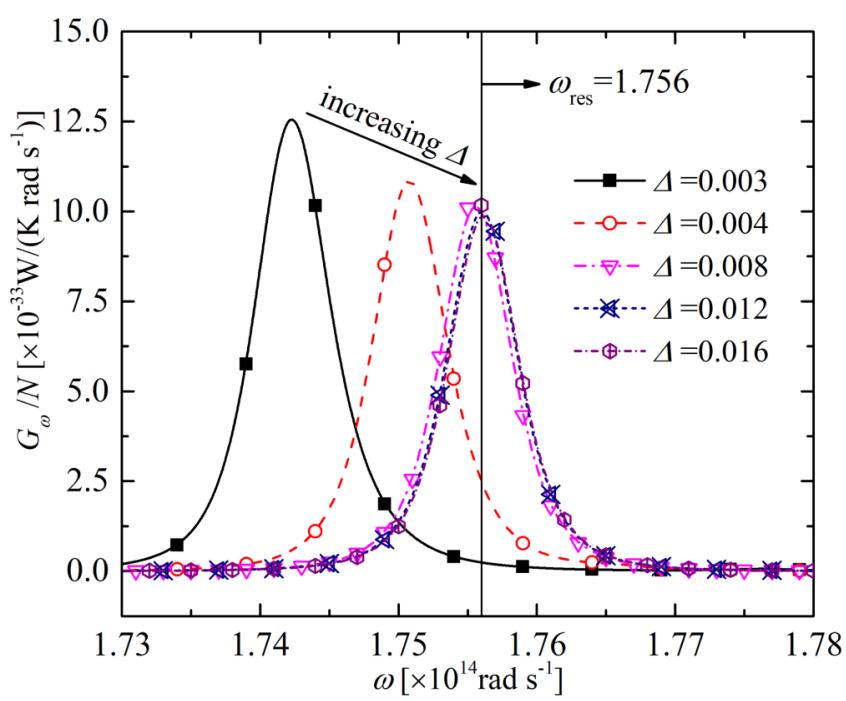

(a) Separation $d=20 \mu \mathrm{m}+2 a$ for the far-field case

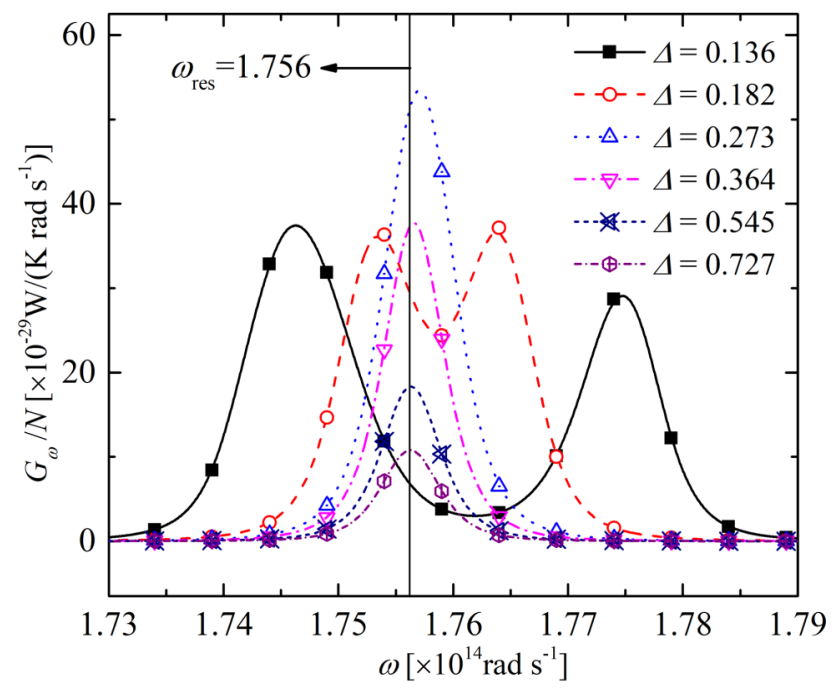

(b) Separation $d=400 \mathrm{~nm}+2 a$ for the near-field case

FIG. 12. The spectral thermal conductance $G_{\omega}$ between two $\mathrm{SiC}$ particle ensembles with various $\Delta=\frac{p}{d}$. Here $N=400, a=20 \mathrm{~nm}$, $T=300 \mathrm{~K}$. Two cases are considered: (a) separation $d=20 \mu \mathrm{m}+$ $2 a$ between the two particle ensembles center to center for the farfield case, $G_{\omega}$ is scaled by the number of nanoparticles from one ensemble $N$, and (b) separation $d=400 \mathrm{~nm}+2 a$ for the near-field case, the $G_{\omega}$ is scaled by the number $N$ of nanoparticles in each ensemble.

the peak value of the spectral thermal conductance increases slightly with the decreasing $\Delta$, which is corresponding to the slightly increasing thermal conductance with decreasing $p$ at a fixed $d(=20.04 \mu \mathrm{m})$ observed in Fig. 6. In the case 1 , the inter-ensemble MBI between the two ensembles is weak and can be neglected safely. However, the many-body interaction inside each of the ensembles (intra-ensemble MBI) is strong due to that nanoparticles from the same ensemble lie in the near field of each other. Therefore, the decreasing pure intra-ensemble MBI may account for the blueshift of the peak frequency of the thermal conductance spectrum, when increasing periodicity $p$.
In addition, in the far-field case, the peak frequency of thermal conductance spectrum between 2D periodic ensembles approaches to the polarizability resonance frequency of the single particle (shown as $\omega_{\text {res }}=1.756 \times 10^{14} \mathrm{rad} \mathrm{s}^{-1}$ in Fig. 12), which satisfies $\operatorname{Re}[\epsilon(\omega)]+2=0$. The spectral thermal conductance between two particle ensembles is the sum of the spectral thermal conductance of all nanoparticle couples, as can be seen from Eq. (6). The nanoparticle ensemble goes more dilute as $\Delta$ increases. Therefore, intra-ensemble MBI goes weaker with increasing $\Delta$. Hence, the thermal conductance spectrum $G_{\omega}(p, d) / N$ between $2 \mathrm{D}$ ensembles is similar to that between two isolated nanoparticles, where the intra-ensemble many-body interaction is negligible.

\section{Case 2: MBI inhibits the RHT $\left\{p \ll \lambda_{T}\right\} \cap\left\{d<\lambda_{T}\right\}$}

In this case, corresponding to the blue region of Fig. 10, the MBI effect inhibits the RHT. When two ensembles are separated by a small distance (near-field case, e.g., $d=400 \mathrm{~nm}$ $\left.+2 a<\lambda_{T}\right)$, the thermal conductance spectrum is shown in Fig. 12(b). The ratio $\psi(p, d)<1$ and MBI effect significantly inhibit the thermal conductance. The peak value of the thermal conductance spectrum increases heavily with decreasing $\Delta$, which also corresponds to that the scaled thermal conductance increases greatly with decreasing $\Delta$ at a small $d(0.44 \mu \mathrm{m})$ observed in Fig. 6. In the case 2, only one peak of the thermal conductance spectrum can be observed for $\Delta \geqslant 0.273$, where intra-ensemble and inter-ensemble MBI effects are weak. For $\Delta=0.136$ and 0.182 , two spectral thermal conductance peaks can be observed, where the intra-ensemble and interensemble MBI effects are strong. The coexisted strong intraensemble and inter-ensemble MBI effects may account for the two peaks of the thermal conductance spectrum between 2D finite-size square-lattice nanoparticle ensembles.

An interesting question is if the phenomenon that thermal conductance spectrum between 2D nanoparticle ensembles in case 2 has two peaks is dependent on the particle distribution or not. Thermal conductance spectrum between 2D nanoparticle ensembles with three different kinds of the particle distribution is shown in Fig. 13: (a) periodic 2D ensemble ( $\Delta=0.182$ ), (b) random $2 \mathrm{D}$ ensemble, and (c) concentricring-configuration $2 \mathrm{D}$ ensemble. Two peaks of the thermal conductance spectrum can be observed for all the three cases, which is independent on the particle distribution. That is to say, the two peaks of thermal conductance spectrum between 2D nanoparticle ensembles with a small separation are due to the many-body interaction and independent on the particle distribution.

It is also worthwhile to mention that the MBI is significantly dependent on the materials. We take the dielectric SiC as an example to analyze the NFRHT in Secs. IV A and IV B. In addition to the materials supporting resonance in the Planck's window (e.g., SiC), we should also pay attention to the materials which do not support resonance in the Planck's window (e.g., Ag). According to our previous works $[38,40]$ for metallic $\mathrm{Ag}$, due to the mismatch between the localized surface resonance wavelength and the thermal wavelength (Planck's window), the MBI on RHT between metallic nanoparticle ensembles (e.g., clusters [38] and nanoparticle chains [40]) can be safely neglected. 


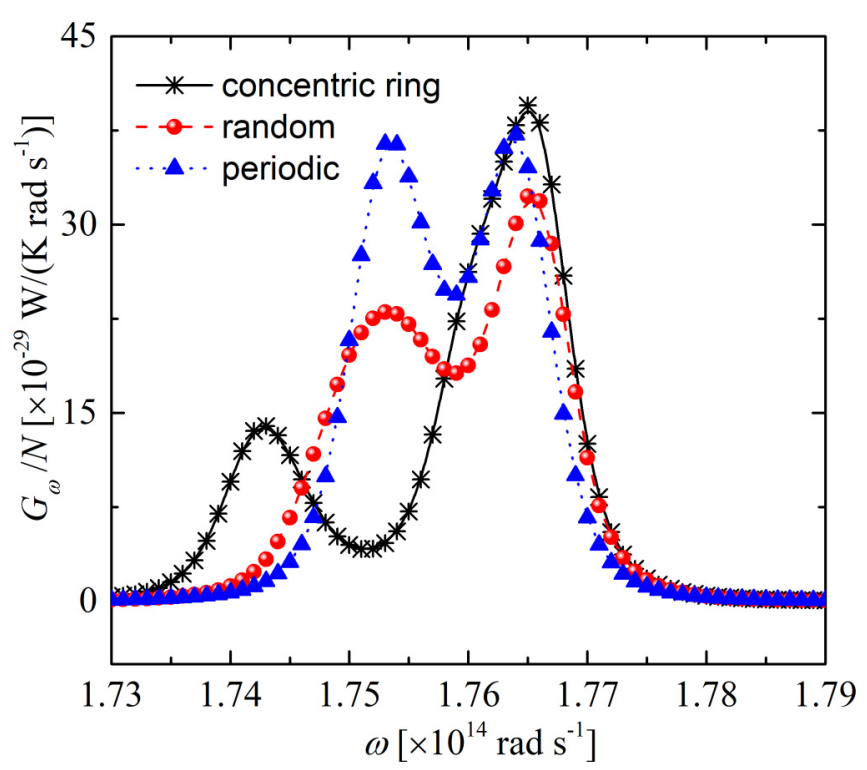

FIG. 13. Thermal conductance spectrum between $2 \mathrm{D}$ ensembles with three kinds of particle distribution in dense regime: (a) periodic $2 \mathrm{D}$ ensemble $(\Delta=0.182)$, (b) random $2 \mathrm{D}$ ensemble, and (c) concentric-ring-configuration $2 \mathrm{D}$ ensemble. $a=20 \mathrm{~nm}, T=$ $300 \mathrm{~K}, N=400$ and separation $d=400 \mathrm{~nm}+2 a$ center to center.

\section{Effect of the phase change on RHT between 2D finite-size square-lattice nanoparticle ensembles}

The optical property of phase-change material below and above transition temperature is quite different from each other. The phase change of material has significant effect on RHT between planar surfaces in both near field and far field [56-60]. However, very few works on the effect of phase change on RHT between 2D nanoparticle ensembles have been reported. The phase change effect will be analyzed in this section.

Thermal conductance between the $2 \mathrm{D}$ periodic nanoparticle ensembles as a function of the separation distance $(d)$ is shown in Fig. 14. The symbols correspond to data obtained at $T=350 \mathrm{~K}$ and the lines correspond to data obtained at $T=$ $300 \mathrm{~K} . N$ is the number of nanoparticles in each ensemble $\mathrm{L}$ and $\mathrm{U}(N=400)$. $p$ is the periodicity of the periodic ensemble $(p=500 \mathrm{~nm})$. Two fitting lines of power law $\left(\sim d^{-2}\right.$ and $\sim d^{-6}$ ) are added for reference.

For the 2D periodic nanoparticle ensembles composed of metallic Ag or dielectric $\mathrm{SiC}$, the thermal conductance at $350 \mathrm{~K}$ is similar to that at $300 \mathrm{~K}$, which is also the case for $2 \mathrm{D}$ phase-change $\mathrm{VO}_{2}$ ensembles with a large separation (the farfield case, larger than $4 \mu \mathrm{m}$ ). However, for 2D periodic phasechange $\mathrm{VO}_{2}$ nanoparticle ensembles with a small separation (the near-field case, smaller than $4 \mu \mathrm{m}$ ), thermal conductance at $300 \mathrm{~K}$ is much higher than that at $350 \mathrm{~K}$, which is an abnormal phenomenon different from the common sense that the thermal conductance often increases with temperature. Phase change of $\mathrm{VO}_{2}$ significantly influences the RHT between 2D nanoparticle ensembles in the near field and has negligible effect on the RHT in the far field.

To understand insight of the abnormal phenomenon (mentioned in the third paragraph) that the thermal conductance

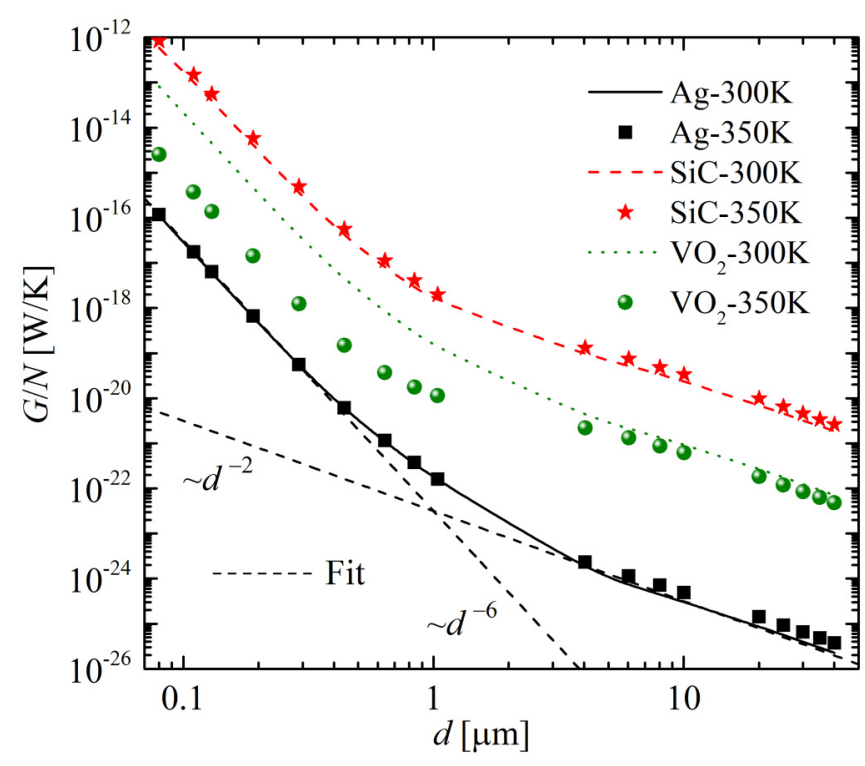

FIG. 14. Thermal conductance between $2 \mathrm{D}$ periodic nanoparticle ensembles composed of metallic $\mathrm{Ag}$, dielectric $\mathrm{SiC}$, and phasechange material $\mathrm{VO}_{2}$. Two different temperatures below and above phase transition temperature of $\mathrm{VO}_{2}$ have been considered, 300 and $350 \mathrm{~K}$, respectively. $a$ is $20 \mathrm{~nm} . N$ is the number of nanoparticles in each ensemble $\mathrm{L}$ and $\mathrm{U}(N=400) . p=500 \mathrm{~nm}$. Two fitting lines of power law $\left(\sim d^{-2}\right.$ and $\left.\sim d^{-6}\right)$ are added for reference.

between two $\mathrm{VO}_{2}$ nanoparticle ensembles decreases with increasing temperature, the spectral thermal conductances between $\mathrm{VO}_{2}$ periodic particle ensembles at 300 and $350 \mathrm{~K}$ are calculated at two different separation distances center to center $(d=40 \mathrm{~nm}+2 a$ and $40 \mu \mathrm{m}+2 a)$, which are shown as Fig. 15.

As can be seen from the thermal conductance spectrum with $d=40 \mathrm{~nm}$ at $300 \mathrm{~K}$, there are two main and obvious

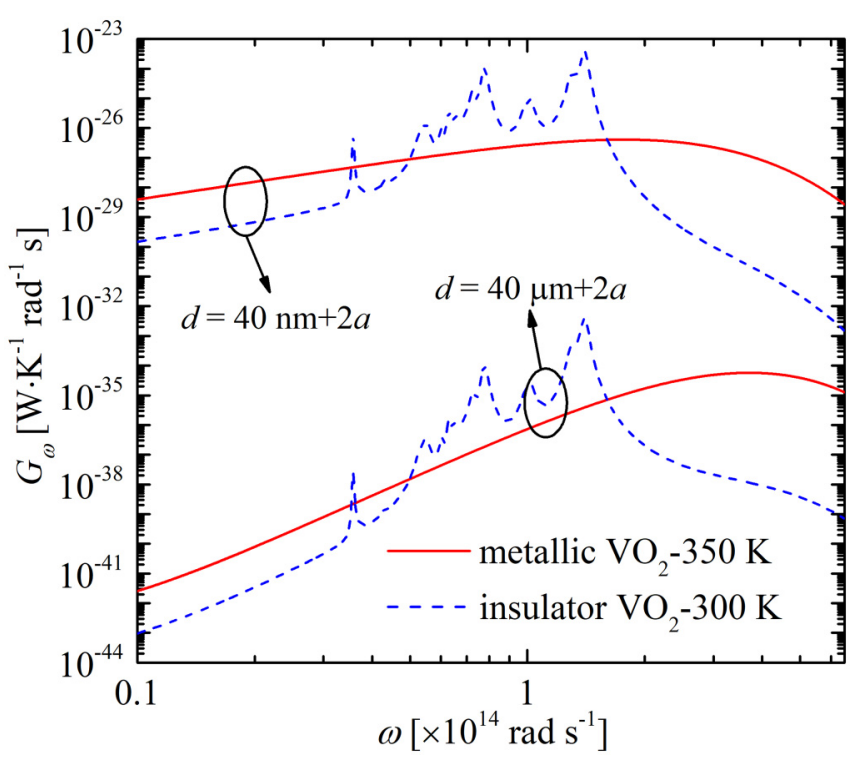

FIG. 15. The spectral thermal conductance between two $2 \mathrm{D} \mathrm{VO}_{2}$ nanoparticle ensembles for $d=40 \mathrm{~nm}+2 a$ and $40 \mu \mathrm{m}+2 a$ at 300 and $350 \mathrm{~K} . a$ is $20 \mathrm{~nm}$. 


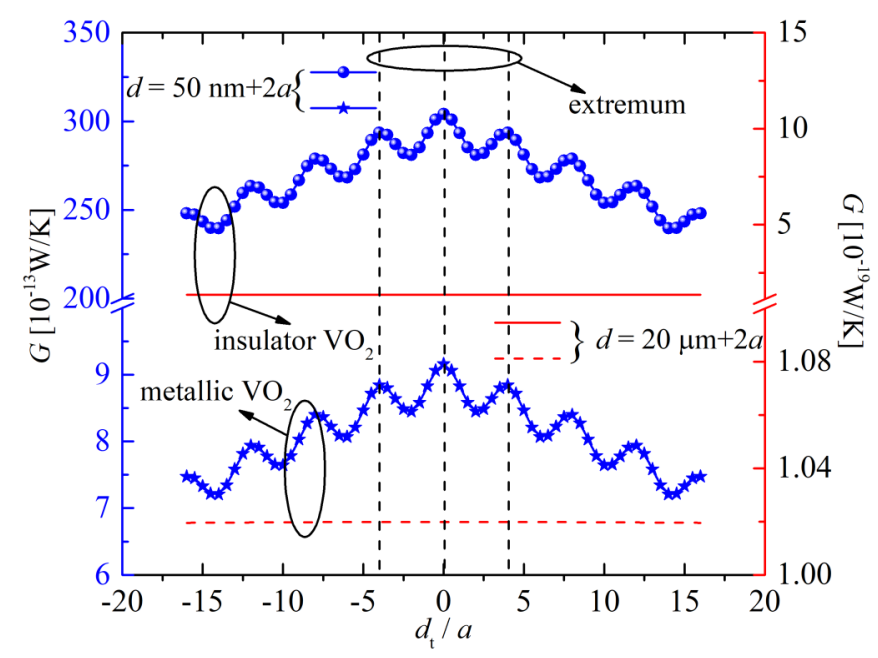

FIG. 16. Thermal conductance between particle ensembles as function of the translation distance of one particle ensemble along its extending direction for several different cases. Red lines are for the data of metallic $\mathrm{VO}_{2}$, while the blue ones are for the insulator $\mathrm{VO}_{2}$. Two different separation distances between the two particle ensembles are considered, $50 \mathrm{~nm}+2 a$ and $20 \mu \mathrm{m}+2 a$, respectively. $a$ is $20 \mathrm{~nm}$. Periodicity of the $2 \mathrm{D}$ periodic nanoparticle ensemble $p$ is $80 \mathrm{~nm} . N=400$.

peaks in the thermal conductance spectrum and the value of spectral thermal conductance around the peaks is much higher than that of the metallic-phase $\mathrm{VO}_{2}$ nanoparticle ensembles at $350 \mathrm{~K}$. The strong localized surface phonon resonance (LSPhR) may account for the high thermal conductance between the insulator-phase $\mathrm{VO}_{2}$ particle ensembles at $300 \mathrm{~K}$. As can be seen in Fig. 3(b), there is a mismatch between the characteristic thermal frequency and polarizability resonance frequency of metallic-phase $\mathrm{VO}_{2}$ nanoparticle, which may account for the lower thermal conductance as compared to that of insulator-phase $\mathrm{VO}_{2}$ nanoparticles. In the far field, e.g., $d=40 \mu \mathrm{m}$, the weak near-field effect of both insulator-phase and metallic-phase $\mathrm{VO}_{2}$ particle ensembles may account for the similar thermal conductance with each other.

\section{Oscillatorylike features of the RHT with translation of the upper 2D finite-size square-lattice nanoparticle ensembles}

A previous investigation on the NFRHT between two gold nanoparticle array layers with an extreme small separation of 1-nm layer edge to layer edge was reported [47], where the multipole contribution to NFRHT has been considered. An interesting oscillatorylike feature of the NFRHT with translation of one array along its extending direction was observed. However, the separation distance between 2D nanoparticle ensembles considered in this work is much larger than that considered in the reported work [47], where the dipole contribution dominates the NFRHT. Thermal conductance between nanoparticle ensembles as a function of the translation distance $d_{\mathrm{t}}$ of one particle ensemble along the translation direction for two different separations $d$ is shown in Fig. 16. Both insulator-phase and metallic-phase $\mathrm{VO}_{2}$ nanoparticles are considered here. Two separation distances $d$ between the $2 \mathrm{D}$ periodic nanoparticle ensembles center to center are

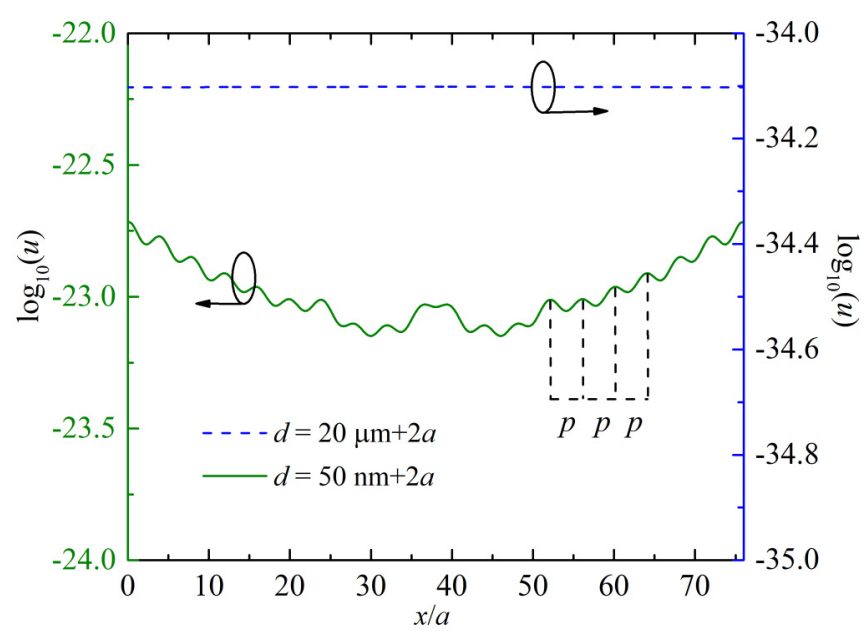

FIG. 17. Energy density along the line with two different separation distances between the line and the lower insulator $\mathrm{VO}_{2}$ nanoparticle ensemble at $300 \mathrm{~K}, 50 \mathrm{~nm}+2 a$, and $20 \mu \mathrm{m}+2 a$, respectively. $p=80 \mathrm{~nm} . N=400$.

considered, $50 \mathrm{~nm}+2 a$ and $20 \mu \mathrm{m}+2 a$, respectively. Nanoparticle radius $a$ is $20 \mathrm{~nm}$. Periodicity of the 2D periodic nanoparticle ensemble $p$ is $80 \mathrm{~nm}$.

When $d=50 \mathrm{~nm}+2 a$, oscillatorylike features of RHT as a function of translation distance $d_{\mathrm{t}}$ of the ensemble $\mathrm{U}$ relative to ensemble $\mathrm{L}$ is shown with star- and circle-symbols lines in Fig. 16. When $d=20 \mu \mathrm{m}+2 a$, no oscillatorylike phenomenon of RHT with translation distance can be observed, shown with solid lines in Fig. 16. From Fig. 16, the oscillatory periodicity is around $80 \mathrm{~nm}(\sim p)$, which is equal to the distance between the neighboring particles in the line parallel to the edge of the particle ensemble and is corresponding to the result observed in the reported work [47]. The oscillatory periodicity of thermal conductance with translation distance $d_{\mathrm{t}}$ is similar to the spatial periodicity of the $2 \mathrm{D}$ periodic nanoparticle ensembles, which may not be an accident. It is noticed that the local energy density distribution has been demonstrated to be very useful to help understanding of physical mechanism of NFRHT, e.g., to analyze the emission of plate [61] and NFRHT among particles in the many-particle system $[33,40]$. To understand insight of the relation between the two kinds of periodicity, i.e., oscillatory periodicity of thermal conductance with translation distance and spatial periodicity $p$ of the $2 \mathrm{D}$ periodic nanoparticle ensemble, analysis of local energy density distribution is conducted. Energy density distribution above the insulator $\mathrm{VO}_{2}$ nanoparticle ensemble is calculated along a line of interest (shown with the yellow line in Fig. 1), which is shown in Fig. 17.

The energy density along the line of interest (shown as the yellow line in Fig. 1) with two different separation distances between the line and the lower insulator $\mathrm{VO}_{2}$ particle ensemble, $d=50 \mathrm{~nm}+2 a$ and $d=20 \mu \mathrm{m}+2 a$, respectively, are shown in Fig. 17. In the far field, i.e., $d=20 \mu \mathrm{m}+2 a$, the energy density stays constant along the line, as shown in Fig. 17. However, the energy density shows an oscillatorylike feature along the line in the near field, i.e., the separation distance $d=50 \mathrm{~nm}+2 a$, of which the oscillatory periodicity 


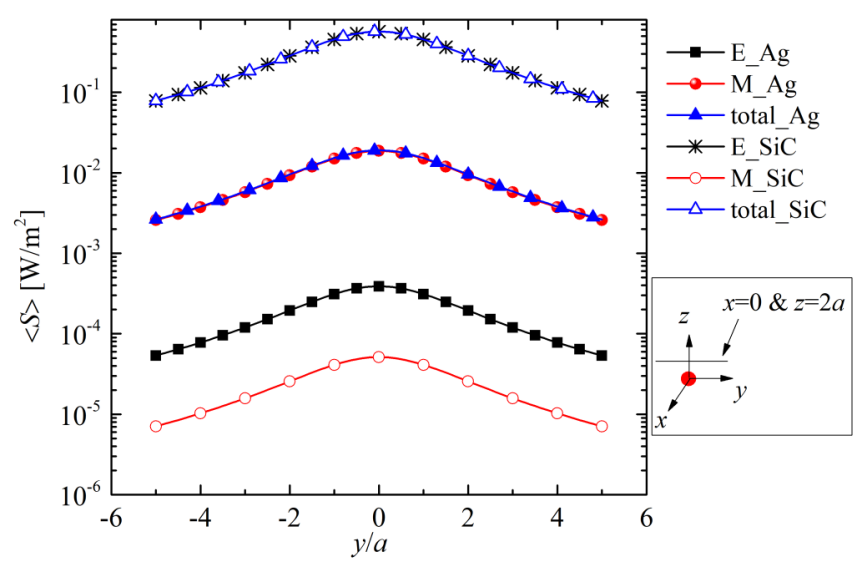

FIG. 18. Poynting vector along the line of interest emitted by a nanoparticle due to electric (E) and magnetic (M) dipole contribution. The investigated physical model is shown in the inset. The nanoparticle is fixed at $(0,0,0)$. Both dielectric $\mathrm{SiC}$ and metallic $\mathrm{Ag}$ nanoparticles are considered. $T$ is $300 \mathrm{~K}$. $a$ is $20 \mathrm{~nm}$.

is also around $80 \mathrm{~nm}(\sim p)$. The oscillatory periodicity of thermal conductance shown in Fig. 16 is corresponding to the oscillatory periodicity of the energy density as shown in Fig. 17. The extremum of the thermal conductance can be reached when the upper ensemble is translated with a distance, of which the value is an integer times of periodicity $p$, as shown in Fig. 16 with the dashed line. The strong near-field effect may account for the oscillatorylike feature of energy density and thermal conductance in the near field $(d=50 \mathrm{~nm}$ $+2 a$ ). With the separation distance increasing, the near-field effect decreases gradually, which accounts for that thermal conductance and energy density have no oscillatorylike feature in the far field.

\section{RADIATIVE THERMAL ENERGY EMITTED BY PERIODIC AND NONPERIODIC 2D NANOPARTICLE ENSEMBLE}

First of all, we compare the electric dipole and magnetic dipole contribution to the radiative thermal energy emitted by a single nanoparticle. Total Poynting vector and Poynting vector contributed by the electric dipole (E) and magnetic dipole (M) emitted by a single nanoparticle is shown in Fig. 18. Both metallic Ag and dielectric nanoparticles are considered. Particle radius $a$ is $20 \mathrm{~nm}$. Temperature $T$ is $300 \mathrm{~K}$. Poynting vector distribution along the line (defined by $x=0$ and $z=$ $2 a$ ) shown with the inset of Fig. 18. Both electric dipole and magnetic dipole contribute to the Poynting vector. For Poynting vector emitted by the dielectric $\mathrm{SiC}$ nanoparticle, the electric dipole contribution is four orders of magnitude larger than that of magnetic dipole contribution. While for Poynting vector emitted by the metallic Ag nanoparticle, the magnetic dipole contribution is two orders of magnitude larger than that of electric dipole contribution. It is worthy to mention that the magnetic dipole contribution dominates the radiative thermal energy emitted by metallic Ag nanoparticle. However, the electric dipole contribution dominates the radiative thermal energy emitted by the dielectric $\mathrm{SiC}$ nanoparticle.

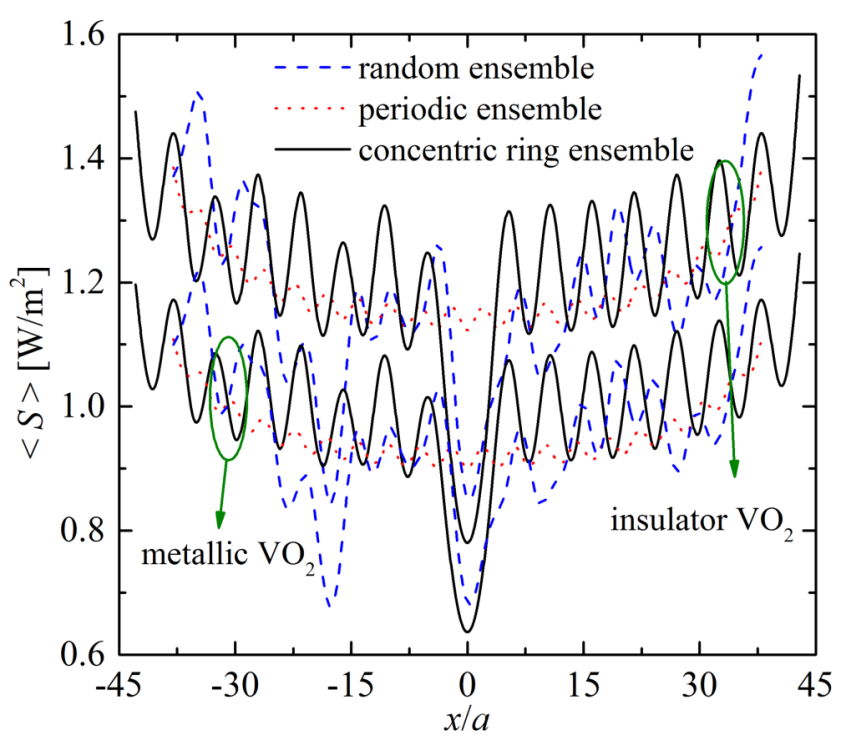

(a) Poynting vector along the line with equation $y=0 \& z=50 \mathrm{~nm}$

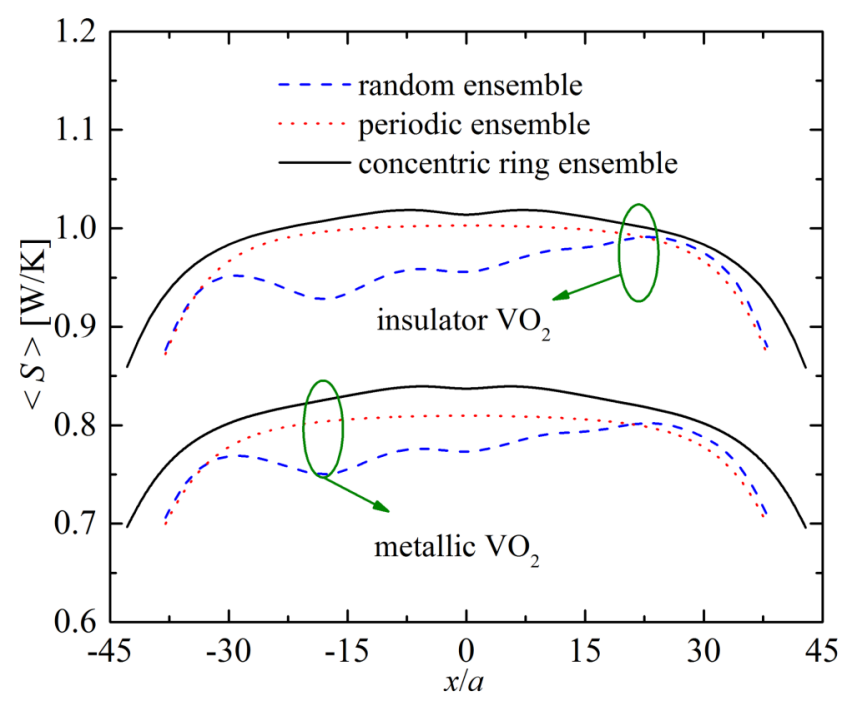

(b) Poynting vector along the line with equation $y=0 \& z=200 \mathrm{~nm}$

FIG. 19. The Poynting vector along the two lines (defined by $y=0$ and $z=50 \mathrm{~nm}$ and $y=0$ and $z=200 \mathrm{~nm}$, respectively) for three different particle ensembles. Both insulator-phase $\mathrm{VO}_{2}$ particle $(300 \mathrm{~K})$ and metallic-phase $\mathrm{VO}_{2}$ particle $(350 \mathrm{~K})$ are considered. $a$ is $20 \mathrm{~nm} . N=400$.

Then, the effect of the nanoparticle distribution on the radiative thermal energy is analyzed. Three different kinds of nanoparticle distributions are considered here: (a) periodic 2D ensemble, (b) random 2D ensemble, and (c) concentric-ringconfiguration 2D ensemble, respectively, as shown in Fig. 2. Considering that the overlap influences the RHT significantly [47], all three different 2D particle ensembles are generated to have similar effective occupied area to each other. Nanoparticle radius $a$ is $20 \mathrm{~nm}$. The Poynting vector along the two lines [defined by (1) $y=0$ and $z=50 \mathrm{~nm}$ and (2) $y=0$ and $z=$ $200 \mathrm{~nm}$, respectively] for all the three different particle ensembles are shown in Figs. 19(a) and 19(b). Both insulator-phase and metallic-phase $\mathrm{VO}_{2}$ nanoparticles are considered. The number of nanoparticles $N$ in each ensemble is 400 . 
When the separation distance between the line and the ensemble is $50 \mathrm{~nm}$, for periodic $2 \mathrm{D}$ ensemble, the Poynting vector shows an oscillatory feature, shown with the red dashed line in Fig. 19(a). For the random 2D ensemble, no obvious regulation can be observed and fluctuation of the Poynting vector also can be observed. However, for concentric-ringconfiguration $2 \mathrm{D}$ ensemble, near the center of the ensemble, the Poynting vector approaches to its minimum, which looks like a deep well. The geometrical configuration of concentric-ring-configuration particle ensemble may account for this focusing and inhibition of the radiative thermal energy. Meanwhile, the periodicity of the structure accounts for the oscillation of the Poynting vector near the periodic and concentric-ring-configuration $2 \mathrm{D}$ ensembles. The disordered nanoparticle distribution in the random 2D ensemble accounts for the irregular distribution of the Poynting vector. Wave effect of the thermally excited evanescent wave accounts for that the radiative thermal energy depends on the geometrical configuration.

When the separation between the line and 2D nanoparticle ensemble increases to $200 \mathrm{~nm}$, as shown in Fig. 19(b), the Poynting vector along the line of interest is quite different from that observed in the case with $50-\mathrm{nm}$ separation. No oscillatory feature of Poynting vector can be observed. The fluctuation of Poynting vector above the random 2D ensemble is much more dramatic than that of the other two 2D nanoparticle ensembles. The near-field effect decreases dramatically with the increasing separation from the source, which accounts for that Poynting vector in the near field is sensitive to the separation distance. Regularly, 2D nanoparticle ensembles, e.g., periodic 2D ensemble and concentricring-configuration $2 \mathrm{D}$ ensembles, emit the radiative thermal energy in a similar way to each other. However, irregularity of the structure results in the irregular distribution and fluctuation of the radiative thermal energy.

From Figs. 19(a) and 19(b), it is noted that the phase change of $\mathrm{VO}_{2}$ only influences the value of the radiative thermal energy and has very weak effect on the distribution regulation of the radiative thermal energy. It is worthwhile to mention that the sign of the curvature of diagrams has changed from Fig. 19(a) to Fig. 19(b). When the separation is small [Fig. 19(a)], the MBI is strong and inhibits the Poynting vector. Therefore, the Poynting near the center of the ensemble is inhibited heavily. However, when the separation is large [Fig. 19(b)], the MBI is weak and each nanoparticle emits energy separately and additionally. The Poynting vector near the center of ensemble is higher than that at other places.

Due to the statistical feature of the random ensemble, we average the Poynting vector over eight realizations of random metallic-phase VO2 nanoparticle ensembles, as shown in Fig. 20. The Poynting vector along the two lines [defined by (1) $y=0$ and $z=50 \mathrm{~nm}$ and (2) $y=0$ and $z=200 \mathrm{~nm}$, respectively] for the random ensembles of eight realizations are also given in Figs. 20(a) and 20(b): $T=350 \mathrm{~K}, a=20 \mathrm{~nm}$. It is shown that the average over the Poynting vector of eight random ensembles reduces the asymmetry. Although each specific configuration of the random ensemble corresponding to a specific emitted Poynting vector distribution, the average of the Poynting vector over a large number realization of random ensembles reduces the asymmetry of the data.

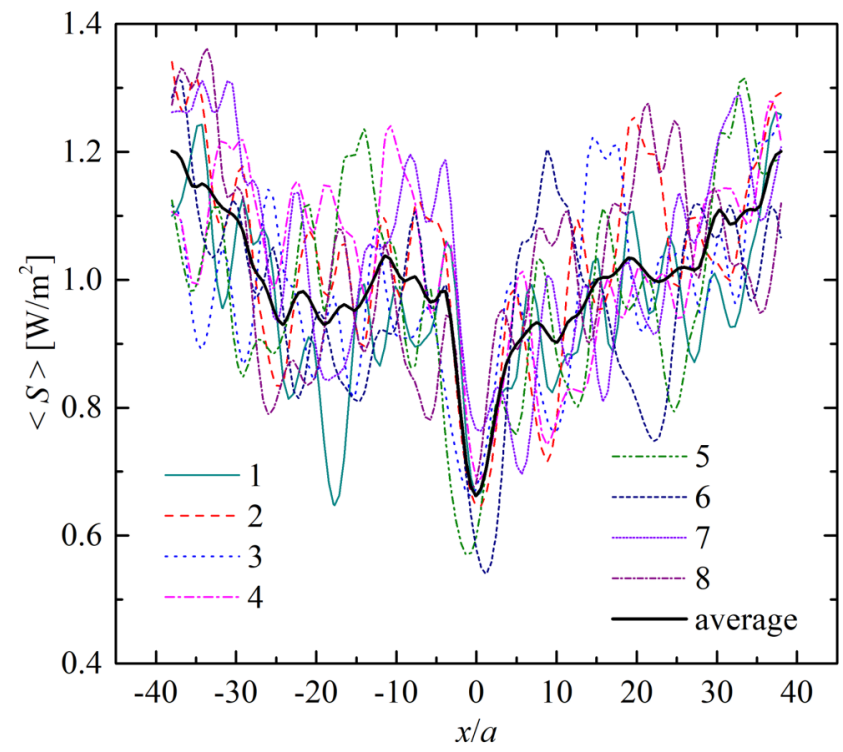

(a) The averaged Poynting vector along the line with equation $y=0 \& z=50 \mathrm{~nm}$

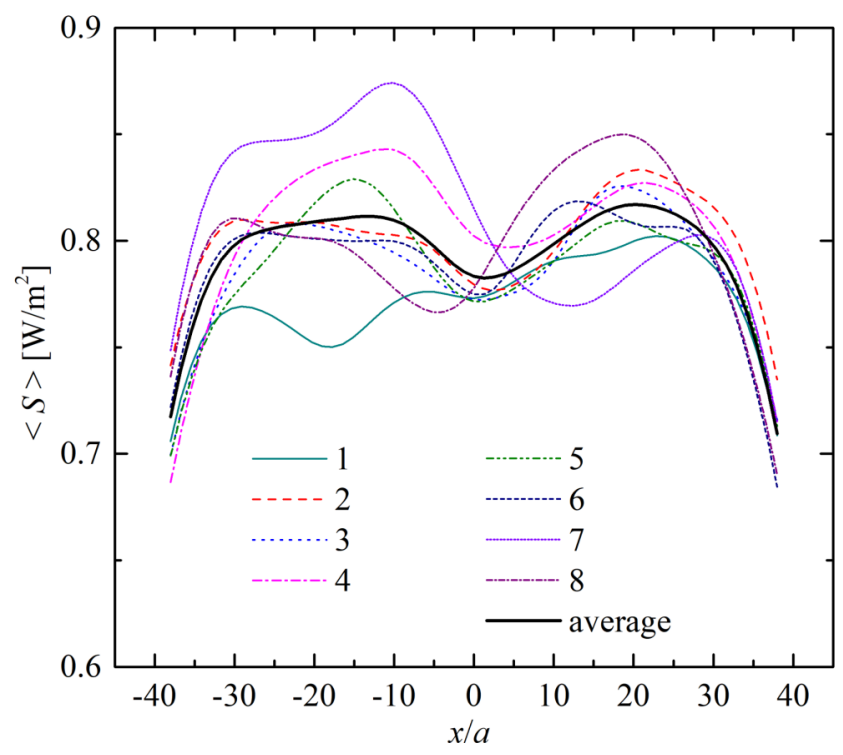

(b) The averaged Poynting vector along the line with equation $y=0 \& z=200 \mathrm{~nm}$

FIG. 20. The averaged Poynting vector over eight random metallic-phase $\mathrm{VO}_{2}$ nanoparticle ensembles along the two lines (defined by $y=0$ and $z=50 \mathrm{~nm}$ and $y=0$ and $z=200 \mathrm{~nm}$, respectively). $T=350 \mathrm{~K}$. $a$ is $20 \mathrm{~nm}$. $N=400$. The lines characterized by the numbers $1-8$ are corresponding to the eight different realizations of the random ensembles.

Considering that the Poynting vector is a vector, it is worth pointing out its direction, which indicates the direction of the radiative thermal energy flow. All components of the Poynting vector emitted by the concentric-ring-configuration 2D ensemble along the line defined by $y=0$ and $z=50 \mathrm{~nm}$ are shown in Fig. 21. The separation distance between the line of interest and the concentric-ring-configuration nanoparticle ensemble is $50 \mathrm{~nm}$. The Poynting vector component in the $y$-axis direction for the whole domain is zero. The radiative thermal energy flow in the plane perpendicular to the 


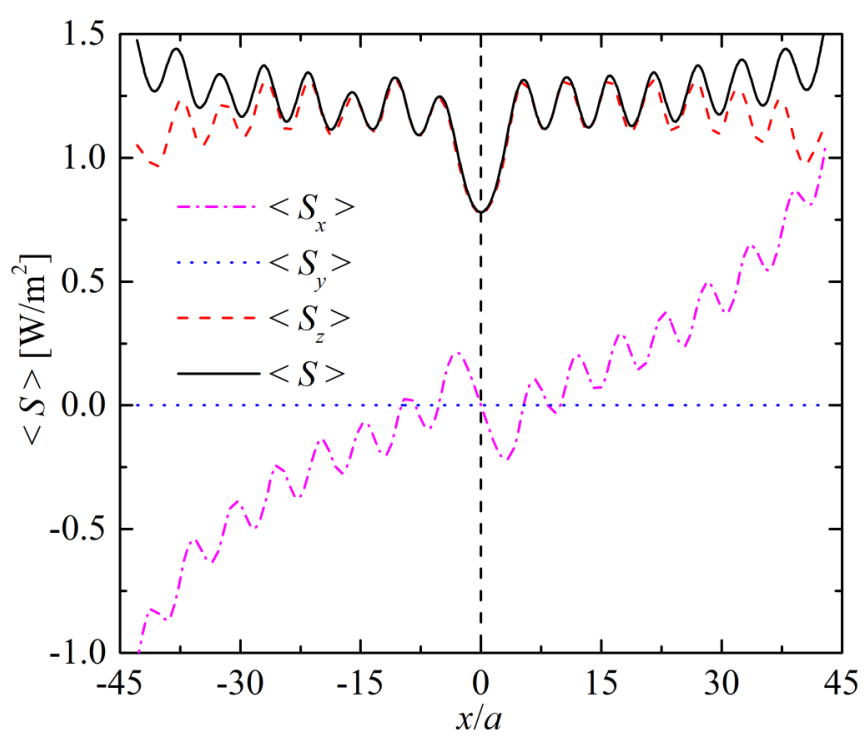

FIG. 21. Components of the Poynting vector emitted by the concentric-ring-configuration 2D ensemble in $x, y$, and $z$ axes of the given Cartesian coordinate system along the line parallel to the $2 \mathrm{D}$ ensemble with 50-nm separation distance. $a=20 \mathrm{~nm} . N=400$.

concentric-ring-configuration $2 \mathrm{D}$ ensemble. The symmetry of the concentric-ring-configuration nanoparticle ensemble geometrical configuration about the line of interest accounts for the radiative thermal energy flow in the plane perpendicular to the $2 \mathrm{D}$ ensemble.

In addition, the radiative thermal energy emitted by the $2 \mathrm{D}$ nanoparticle ensemble is sensitive to the separation from the ensemble. To help for the understanding of this separationdependent radiative thermal energy, the energy density along the lines of interest (shown in Fig. 1) parallel to the 2D ensemble with nine different separations is shown in Fig. 22. When the separation distance between the line and 2D nanoparticle ensemble is larger than $500 \mathrm{~nm}$, the energy density does not vary from place to place any more. However, when the

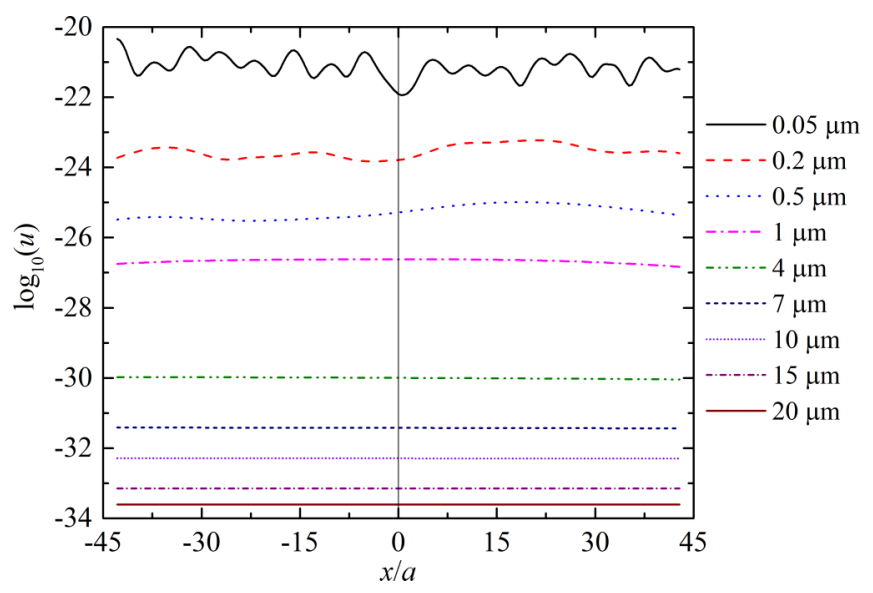

FIG. 22. Energy density along the line above the concentricring-configuration particle ensemble with nine different separation distances: $0.05,0.2,0.5,1,4,7,10,15$, and $20 \mu \mathrm{m}$, respectively. $N=400$. separation distance is less than $500 \mathrm{~nm}$, the energy density oscillates along the line and is symmetrical in general about the center of the line, which can be attributed to the symmetry of the geometrical configuration of the 2D concentric-ringconfiguration nanoparticle ensemble. Energy density is also dependent on the separation from the $2 \mathrm{D}$ ensemble, which is corresponding to that observed for the Poynting vector. The separation dependent near-field effect accounts for both the separation-dependent observation of the Poynting vector and energy density.

\section{CONCLUSION}

Radiative heat transfer (RHT) and radiative thermal energy concerning 2D nanoparticle ensembles is investigated in both the near field and far field by means of the CEMD approach and the proposed formulas of Poynting vector based on the framework of many-body radiative heat transfer theory. Asymptotic regimes of RHT between 2D finite-size squarelattice nanoparticle ensembles were summarized in Table I and the regime map Fig. 5. Four regimes and their corresponding thermal conductance formulas were given explicitly: (a) MBI regime $\left\{p \ll \lambda_{T}\right\}$, (b) non-MBI regime, (c) rarefied regime, and (d) dense regime, respectively. In the rarefied regime, dense regime, and non-MBI regime, the thermal conductance formulas can be simplified as compared to the general formula.

According to the value of the parameter $\psi(p, d)$, we numerically identified the different asymptotic regimes in detail. In the MBI regime $p \ll \lambda_{T}$, MBI effects manifest themselves in different ways, depending on the separation $d$ significantly. In the MBI regime, both the value of the thermal conductance and shape of the thermal conductance spectrum $G_{\omega}$ can be influenced by the MBI effects. We considered two typical cases to analyze MBI effects on the spectrum $G_{\omega}$ : case 1 (MBI enhances the RHT, $\left.\left\{p \ll \lambda_{T}\right\} \cap\left\{d>\lambda_{T}\right\}\right)$ and case 2 (MBI inhibits the RHT $\left.\left\{p \ll \lambda_{T}\right\} \cap\left\{d<\lambda_{T}\right\}\right)$. In the case 1 , the decreasing pure intra-ensemble MBI accounts for the blueshift of the peak of the spectrum $G_{\omega}$, and has a slight enhancement effect on the thermal conductance. In the case 2, the strong coexisted intra-ensemble and inter-ensemble MBI account for inhibition of RHT and the two peaks of the spectrum $G_{\omega}$ as compared to that of two isolated nanoparticles, which also has been demonstrated independent of the nanoparticle distribution.

The thermal conductance between 2D insulator-phase $\mathrm{VO}_{2}$ nanoparticle ensembles with a small separation is much larger than that of the metallic-phase $\mathrm{VO}_{2}$ nanoparticle ensembles due to the strong coupling in the insulator-phase $\mathrm{VO}_{2}$ nanoparticle ensembles. However, this phase-change effect is negligible when the separation is large. An oscillatorylike feature of thermal conductance between 2D finite-size square-lattice nanoparticle ensembles with translation of the upper ensemble relative to the lower ensemble and thermally excited energy density distribution in the near field is observed, which is negligible in the far field. The strong near-field effect accounts for the oscillatory phenomenon of the thermal conductance and energy density.

In addition, the formulas of the Poynting vector to evaluate the radiative thermal energy taking both electric and magnetic 
dipole contribution into consideration are given, which is an extension of the recent work [46] and is applicable for not only dielectric but also metallic nanoparticle ensemble. It is the magnetic dipole contribution that dominates the radiative thermal energy emitted by the metallic nanoparticle. However, the electric dipole contribution dominates the radiative thermal energy emitted by the dielectric nanoparticle. The radiative thermal energy emitted by the $2 \mathrm{D}$ nanoparticle ensemble is sensitive to the particle distribution and the distance away from the ensemble. The RTE emitted by $2 \mathrm{D}$ concentric-ring- configuration nanoparticle ensemble has an inhibition feature near the ensemble center of the ensemble.

\section{ACKNOWLEDGMENTS}

The support of this work by the National Natural Science Foundation of China (Grant No. 51976045) is gratefully acknowledged. M.A. acknowledges support from the Institute Universitaire de France, Paris, France (UE). M.G.L. also thanks for the support from China Scholarship Council (Grant No. 201906120208).

\section{APPENDIX: GREEN'S FUNCTION IN MANY-PARTICLE SYSTEM}

In this Appendix, following the previous paper [38], we provide the details about how to calculate the many-body Green's function $G_{i j}^{\mu \nu}$ needed to evaluate the RHT of Eqs. (1) and (2), and $G^{\nu \tau}\left(\mathbf{r}, \mathbf{r}_{i}\right)$ needed to evaluate the RTE of Eqs. (14) and (15). Let us start by defining the Green's function in free space:

$$
\begin{gathered}
G_{0}^{E E}(\mathbf{r})=\frac{e^{i k r}}{4 \pi r}\left[\left(1+\frac{i k r-1}{k^{2} r^{2}}\right) \mathbb{I}_{3}+\frac{3-3 i k r-k^{2} r^{2}}{k^{2} r^{2}} \hat{\mathbf{r}} \otimes \hat{\mathbf{r}}\right], \\
G_{0}^{M E}(\mathbf{r})=\frac{e^{i k r}}{4 \pi r}\left(1-\frac{1}{i k r}\right)\left(\begin{array}{ccc}
0 & -\hat{r}_{z} & \hat{r}_{y} \\
\hat{r}_{z} & 0 & -\hat{r}_{x} \\
-\hat{r}_{y} & \hat{r}_{x} & 0
\end{array}\right),
\end{gathered}
$$

where $\mathbb{I}_{3}$ is a $3 \times 3$ identity matrix, $r$ is the magnitude of the separation vector $\mathbf{r}=\mathbf{r}_{f}-\mathbf{r}_{s}$ between the source point $\mathbf{r}_{s}$ and field point $\mathbf{r}_{f}, \hat{\mathbf{r}}$ is the unit vector $\mathbf{r} / r$ and $\hat{r}_{\nu=x, y, z}$ denotes its three Cartesian components, $\otimes$ denotes outer product of vectors. $G_{0}^{E M}(\mathbf{r})=-G_{0}^{E M}(\mathbf{r})$ and $G_{0}^{M M}(\mathbf{r})=G_{0}^{E E}(\mathbf{r})$. The Green's function in free space can be written in a compact form as

$$
\mathbb{G}_{0, i j}=\left(\begin{array}{cc}
\mu_{0} \omega^{2} G_{0, i j}^{E E} & \mu_{0} \omega G_{0, i j}^{E M} \\
k \omega G_{0, i j}^{M E} & k^{2} G_{0, i j}^{M M}
\end{array}\right),
$$

where $G_{0, i j}^{\mu \nu} \equiv G_{0}^{\mu \nu}\left(\mathbf{r}_{i}-\mathbf{r}_{j}\right)$. Also, the many-body Green's function $G_{i j}^{\mu \nu}$ we are looking for can be written by exploiting the compact form

$$
\mathbb{G}_{i j}=\left(\begin{array}{cc}
\mu_{0} \omega^{2} G_{i j}^{E E} & \mu_{0} \omega G_{i j}^{E M} \\
k \omega G_{i j}^{M E} & k^{2} G_{i j}^{M M}
\end{array}\right) .
$$

The function $\mathbb{G}_{i j}$ is the solution of the equation

$$
\left(\begin{array}{cccc}
0 & \mathbb{G}_{12} & \cdots & \mathbb{G}_{1 N} \\
\mathbb{G}_{21} & 0 & \ddots & \vdots \\
\vdots & \vdots & \ddots & \mathbb{G}_{(N-1) N} \\
\mathbb{G}_{N 1} & \mathbb{G}_{N 2} & \cdots & 0
\end{array}\right)=\left(\begin{array}{cccc}
0 & \mathbb{G}_{0,12} & \cdots & \mathbb{G}_{0,1 N} \\
\mathbb{G}_{0,21} & 0 & \ddots & \vdots \\
\vdots & \vdots & \ddots & \mathbb{G}_{0,(N-1) N} \\
\mathbb{G}_{0, N 1} & \mathbb{G}_{0, N 2} & \cdots & 0
\end{array}\right) \mathbb{A}^{-1},
$$

where the matrix $\mathbb{A}$ including many-body interaction is defined as

$$
\mathbb{A}=\mathbb{I}_{6 N}-\left(\begin{array}{cccc}
0 & \boldsymbol{\alpha}_{1} \mathbb{G}_{0,12} & \cdots & \boldsymbol{\alpha}_{1} \mathbb{G}_{0,1 N} \\
\boldsymbol{\alpha}_{2} \mathbb{G}_{0,21} & 0 & \ddots & \vdots \\
\vdots & \vdots & \ddots & \boldsymbol{\alpha}_{N-1} \mathbb{G}_{0,(N-1) N} \\
\boldsymbol{\alpha}_{N} \mathbb{G}_{0, N 1} & \cdots & \boldsymbol{\alpha}_{N} \mathbb{G}_{0, N(N-1)} & 0
\end{array}\right),
$$

with $\boldsymbol{\alpha}_{i}$ defined as

$$
\boldsymbol{\alpha}_{i}=\left(\begin{array}{cc}
\epsilon_{0} \alpha_{E}^{i} \mathbb{I}_{3} & 0 \\
0 & \alpha_{H}^{i} \mathbb{I}_{3}
\end{array}\right) .
$$

To finish, the many-body Green's function $G^{\nu \tau}\left(\mathbf{r}, \mathbf{r}_{i}\right) \equiv G_{\mathbf{r r}_{i}}^{\nu \tau}$ we are looking for to calculate the RTE can also be written in the compact form

$$
\mathbb{G}_{\mathbf{r r}_{i}}=\left(\begin{array}{cc}
\mu_{0} \omega^{2} G_{\mathbf{r r}_{i}}^{E E} & \mu_{0} \omega G_{\mathbf{r r}_{i}}^{E M} \\
k \omega G_{\mathbf{r r}_{i}}^{M E} & k^{2} G_{\mathbf{r r}_{i}}^{M M}
\end{array}\right) .
$$


The function $\mathbb{G}_{\mathbf{r r}_{1}}$ is the solution of the equation

$$
\left(\mathbb{G}_{\mathbf{r r}_{1}}, \mathbb{G}_{\mathbf{r r}_{2}}, \ldots, \mathbb{G}_{\mathbf{r r}_{N}}\right)=\left(\mathbb{G}_{0, \mathbf{r r}_{1}}, \mathbb{G}_{0, \mathbf{r r}_{2}}, \ldots, \mathbb{G}_{0, \mathbf{r r}_{N}}\right) \mathbb{A}^{-1},
$$

where the matrix $\mathbb{A}$ can be found in Eq. (A6) and the source term $\mathbb{G}_{0, \mathbf{r r}_{i}}$ is defined as

$$
\mathbb{G}_{0, \mathbf{r r}_{i}}=\left(\begin{array}{cc}
\mu_{0} \omega^{2} G_{0, \mathbf{r} r_{i}}^{E E} & \mu_{0} \omega G_{0, \mathbf{r r}_{i}}^{E M} \\
k \omega G_{0, \mathbf{r r}_{i}}^{M E} & k^{2} G_{0, \mathbf{r r}_{i}}^{M M}
\end{array}\right),
$$

where $G_{0, \mathbf{r r}_{i}}^{\mu v} \equiv G_{0}^{\mu v}\left(\mathbf{r}-\mathbf{r}_{i}\right)$

[1] S. M. Rytov, Y. A. Kravtsov, and V. I. Tatarskii, Priniciples of Statistical Radiophysics, Vol. 3 (Springer, Berlin, 1989).

[2] D. Polder and M. Van Hove, Theory of radiative heat transfer between closely spaced bodies, Phys. Rev. B 4, 3303 (1971).

[3] J. J. Loomis and H. J. Maris, Theory of heat transfer by evanescent electromagnetic waves, Phys. Rev. B 50, 18517 (1994).

[4] R. Carminati and J.-J. Greffet, Near-Field Effects in Spatial Coherence of Thermal Sources, Phys. Rev. Lett. 82, 1660 (1999).

[5] A. V. Shchegrov, K. Joulain, R. Carminati, and J.-J. Greffet, Near-Field Spectral Effects Due to Electromagnetic Surface Excitations, Phys. Rev. Lett. 85, 1548 (2000).

[6] A. I. Volokitin and B. N. J. Persson, Radiative heat transfer between nanostructures, Phys. Rev. B 63, 205404 (2001).

[7] A. Narayanaswamy and G. Chen, Surface modes for near field thermophotovoltaics, Appl. Phys. Lett. 82, 3544 (2003).

[8] A. I. Volokitin and B. N. J. Persson, Resonant photon tunneling enhancement of the radiative heat transfer, Phys. Rev. B 69, 045417 (2004).

[9] P.-O. Chapuis, M. Laroche, S. Volz, and J.-J. Greffet, Radiative heat transfer between metallic nanoparticles, Appl. Phys. Lett. 92, 201906 (2008).

[10] A. Manjavacas and F. J. García de Abajo, Radiative heat transfer between neighboring particles, Phys. Rev. B 86, 075466 (2012).

[11] M. Nikbakht, Radiative heat transfer between core-shell nanoparticles, J. Quant. Spectrosc. Radiat. Transfer 221, 164 (2018).

[12] A. Narayanaswamy and G. Chen, Thermal near-field radiative transfer between two spheres, Phys. Rev. B 77, 075125 (2008).

[13] P.-O. Chapuis, M. Laroche, S. Volz, and J.-J. Greffet, Nearfield induction heating of metallic nanoparticles due to infrared magnetic dipole contribution, Phys. Rev. B 77, 125402 (2008).

[14] R. Messina, S.-A. Biehs, and P. Ben-Abdallah, Surfacemode-assisted amplification of radiative heat transfer between nanoparticles, Phys. Rev. B 97, 165437 (2018).

[15] J. Dong, J. M. Zhao, and L. H. Liu, Long-distance near-field energy transport via propagating surface waves, Phys. Rev. B 97, 075422 (2018).

[16] Y. Zhang, M. Antezza, H. L. Yi, and H. P. Tan, Metasurfacemediated anisotropic radiative heat transfer between nanoparticles, Phys. Rev. B 100, 085426 (2019).

[17] M. J. He, H. Qi, Y. T. Ren, Y. J. Zhao, and M. Antezza, Graphene-based thermal repeater, Appl. Phys. Lett. 115, 263101 (2019).

[18] Y. Zhang, H. L. Yi, H. P. Tan, and M. Antezza, Giant resonant radiative heat transfer between nanoparticles, Phys. Rev. B 100, 134305 (2019).
[19] R. Messina and M. Antezza, Casimir-lifshitz force out of thermal equilibrium and heat transfer between arbitrary bodies, Europhys. Lett. 95, 61002 (2011).

[20] R. Messina and M. Antezza, Three-body radiative heat transfer and casimir-lifshitz force out of thermal equilibrium for arbitrary bodies, Phys. Rev. A 89, 052104 (2014).

[21] I. Latella, P. Ben-Abdallah, S.-A. Biehs, M. Antezza, and R. Messina, Radiative heat transfer and nonequilibrium casimirlifshitz force in many-body systems with planar geometry, Phys. Rev. B 95, 205404 (2017).

[22] R. S. Ottens, V. Quetschke, S. Wise, A. A. Alemi, R. Lundock, G. Mueller, D. H. Reitze, D. B. Tanner, and B. F. Whiting, NearField Radiative Heat Transfer between Macroscopic Planar Surfaces, Phys. Rev. Lett. 107, 014301 (2011).

[23] M. Lim, S. S. Lee, and B. J. Lee, Near-field thermal radiation between doped silicon plates at nanoscale gaps, Phys. Rev. B 91, 195136 (2015).

[24] J. I. Watjen, B. Zhao, and Z. M. Zhang, Near-field radiative heat transfer between doped-si parallel plates separated by a spacing down to 200 nm, Appl. Phys. Lett. 109203112 (2016).

[25] M. Ghashami, H. Geng, T. Kim, N. Iacopino, S. K. Cho, and K. Park, Precision Measurement of Phonon-Polaritonic Near-Field Energy Transfer between Macroscale Planar Structures Under Large Thermal Gradients, Phys. Rev. Lett. 120, 175901 (2018).

[26] J. DeSutter, L. Tang, and M. Francoeur, A near-field radiative heat transfer device, Nat. Nanotechnol. 14, 751 (2019).

[27] S. Shen, A. Narayanaswamy, and G. Chen, Surface phonon polaritons mediated energy transfer between nanoscale gaps, Nano Lett. 9, 2909 (2009).

[28] E. Rousseau, A. Siria, G. Jourdan, S. Volz, F. Comin, J. Chevrier, and J.-J. Greffet, Radiative heat transfer at the nanoscale, Nat. Photonics 3, 514 (2009).

[29] B. Song, Y. Ganjeh, S. Sadat, D. Thompson, A. Fiorino, V. Fernández-Hurtado, J. Feist, F. J. Garcia-Vidal, J. C. Cuevas, P. Reddy, and E. Meyhofer, Enhancement of near-field radiative heat transfer using polar dielectric thin films, Nat. Nanotechnol. 10, 253 (2015).

[30] P. Ben-Abdallah, R. Messina, S.-A. Biehs, M. Tschikin, K. Joulain, and C. Henkel, Heat Superdiffusion in Plasmonic Nanostructure Networks, Phys. Rev. Lett. 111, 174301 (2013).

[31] E. Tervo, Z. M. Zhang, and B. Cola, Collective near-field thermal emission from polaritonic nanoparticle arrays, Phys. Rev. Mater. 1, 015201 (2017).

[32] P. Ben-Abdallah, S.-A. Biehs, and K. Joulain, Many-Body Radiative Heat Transfer Theory, Phys. Rev. Lett. 107, 114301 (2011). 
[33] J. Dong, J. M. Zhao, and L. H. Liu, Radiative heat transfer in many-body systems: Coupled electric and magnetic dipole approach, Phys. Rev. B 95, 125411 (2017).

[34] M. Krüger, G. Bimonte, T. Emig, and M. Kardar, Trace formulas for nonequilibrium casimir interactions, heat radiation, and heat transfer for arbitrary objects, Phys. Rev. B 86, 115423 (2012).

[35] B. Müller, R. Incardone, M. Antezza, T. Emig, and M. Krüger, Many-body heat radiation and heat transfer in the presence of a nonabsorbing background medium, Phys. Rev. B 95, 085413 (2017).

[36] B. Czapla and A. Narayanaswamy, Thermal radiative energy exchange between a closely-spaced linear chain of spheres and its environment, J. Quant. Spectrosc. Radiat. Transfer 227, 4 (2019).

[37] J. Dong, J. M. Zhao, and L. H. Liu, Near-field radiative heat transfer between clusters of dielectric nanoparticles, J. Quant. Spectrosc. Radiat. Transfer 197, 114 (2017).

[38] M. G. Luo, J. Dong, J. M. Zhao, L. H. Liu, and M. Antezza, Radiative heat transfer between metallic nanoparticle clusters in both near field and far field, Phys. Rev. B 99, 134207 (2019).

[39] J. Chen, C. Y. Zhao, and B. X. Wang, Near-field thermal radiative transfer in assembled spherical systems composed of core-shell nanoparticles, J. Quant. Spectrosc. Radiat. Transfer 219, 304 (2018).

[40] M. G. Luo, J. M. Zhao, and L. H. Liu, Near-field radiative heat transfer in a chain of nanoparticles with another chain in proximity, J. Quant. Spectrosc. Radiat. Transfer 243, 106801 (2020).

[41] I. Latella, S.-A. Biehs, R. Messina, A. W. Rodriguez, and P. Ben-Abdallah, Ballistic near-field heat transport in dense manybody systems, Phys. Rev. B 97, 035423 (2018).

[42] E. Tervo, M. Francoeur, B. Cola, and Z. M. Zhang, Thermal radiation in systems of many dipoles, Phys. Rev. B 100, 205422 (2019)

[43] P. Ben-Abdallah, K. Joulain, J. Drevillon, and C. Le Goff, Heat transport through plasmonic interactions in closely spaced metallic nanoparticle chains, Phys. Rev. B 77, 075417 (2008).

[44] E. J. Tervo, O. S. Adewuyi, J. S. Hammonds, and B. A. Cola, High thermal conductivity in polaritonic $\mathrm{SiO}_{2}$ nanoparticle beds, Mater. Horiz. 3, 434 (2016).

[45] C. Kathmann, R. Messina, P. Ben-Abdallah, and S.-A. Biehs, Limitations of kinetic theory to describe near-field heat exchanges in many-body systems, Phys. Rev. B 98, 115434 (2018).

[46] P. Ben-Abdallah, Multitip Near-Field Scanning Thermal Microscopy, Phys. Rev. Lett. 123, 264301 (2019).
[47] A. D. Phan, T.-L. Phan, and L. M. Woods, Near-field heat transfer between gold nanoparticle arrays, J. Appl. Phys. 114, 214306 (2013).

[48] M. Nikbakht, Radiative heat transfer in fractal structures, Phys. Rev. B 96, 125436 (2017).

[49] M. Francoeur and M. P. Mengüç, Role of fluctuational electrodynamics in near-field radiative heat transfer, J. Quant. Spectrosc. Radiat. Transfer 109, 280 (2008).

[50] M. A. Ordal, R. J. Bell, R. W. Alexander, L. L. Long, and M. R. Querry, Optical properties of fourteen metals in the infrared and far infrared: Al, Co, Cu, Au, Fe, Pb, Mo, Ni, Pd, Pt, Ag, Ti, V, and W, Appl. Opt. 24, 4493 (1985).

[51] E. Palik, Handbook of Optical Constants of Solids (Academic, New York, 1998).

[52] A. S. Barker, H. W. Verleur, and H. J. Guggenheim, Infrared Optical Properties of Vanadium Dioxide above and Below the Transition Temperature, Phys. Rev. Lett. 17, 1286 (1966).

[53] M. Quinten, Optical Properties of Nanoparticle Systems: Mie and Beyond (Wiley, Hoboken, NJ, 2010).

[54] Y. H. Wang and J. Z. Wu, Radiative heat transfer between nanoparticles enhanced by intermediate particle, AIP Adv. 6, 025104 (2016).

[55] J. L. Song, Q. Cheng, Z. X. Luo, X. P. Zhou, and Z. M. Zhang, Modulation and splitting of three-body radiative heat flux via graphene/sic core-shell nanoparticles, Int. J Heat Mass Transfer 140, 80 (2019).

[56] S. Taylor, Y. Yang, and L. P. Wang, Vanadium dioxide based fabry-perot emitter for dynamic radiative cooling applications, J. Quant. Spectrosc. Radiat. Transfer 197, 76 (2017).

[57] Z. H. Zheng, X. L. Liu, A. Wang, and Y. M. Xuan, Grapheneassisted near-field radiative thermal rectifier based on phase transition of vanadium dioxide $\left(\mathrm{VO}_{2}\right)$, Int. J. Heat Mass Transfer 109, 63 (2017).

[58] C. L. Gomez-Heredia, J. A. Ramirez-Rincon, J. OrdonezMiranda, O. Ares, J. J. Alvarado-Gil, C. Champeaux, F. DumasBouchiat, Y. Ezzahri, and K. Joulain, Thermal hysteresis measurement of the $\mathrm{VO}_{2}$ emissivity and its application in thermal rectification, Sci. Rep. 8, 8479 (2018).

[59] J. Ordonez-Miranda, Y. Ezzahri, J. A. Tiburcio-Moreno, K. Joulain, and J. Drevillon, Radiative Thermal Memristor, Phys. Rev. Lett. 123, 025901 (2019).

[60] D. Szilard, W. J. M. Kort-Kamp, F. S. S. Rosa, F. A. Pinheiro, and C. Farina, Hysteresis in the spontaneous emission induced by $\mathrm{VO}_{2}$ phase change, J. Opt. Soc. Am. B 36, C46 (2019).

[61] K. Joulain, J.-P. Mulet, F. Marquier, R. Carminati, and J.-J. Greffet, Surface electromagnetic waves thermally excited: Radiative heat transfer, coherence properties and casimir forces revisited in the near field, Surf. Sci. Rep. 57, 59 (2005). 